\title{
Emergence of Hidden Attractors through the Rupture of Heteroclinic-Like Orbits of Switched Systems with Self-Excited Attractors
}

\author{
R. J. Escalante-González $\mathbb{i D}^{1}$ and Eric Campos $\mathbb{D}^{2}$ \\ ${ }^{1}$ Electrical, Electronic and Mechatronics Department, Technological Institute of San Luis Potosí, Tecnológico Avenue, \\ Soledad de Graciano Sánchez, San Luis Potosí 78437, Mexico \\ ${ }^{2}$ División de Control y Sistemas Dinámicos, Instituto Potosino de Investigación Científica y Tecnológica (IPICYT), \\ Camino a La Presa San José 2055, Lomas 4 Sección 78216, San Luis Potosí, S.L.P., Mexico
}

Correspondence should be addressed to R. J. Escalante-González; rodolfo.escalante@outlook.com

Received 10 February 2021; Revised 6 July 2021; Accepted 15 August 2021; Published 6 September 2021

Academic Editor: Xianggui Guo

Copyright (C) 2021 R. J. Escalante-González and Eric Campos. This is an open access article distributed under the Creative Commons Attribution License, which permits unrestricted use, distribution, and reproduction in any medium, provided the original work is properly cited.

\begin{abstract}
This work is dedicated to the study of an approach that allows the generation of hidden attractors based on a class of piecewiselinear (PWL) systems. The systems produced with the approach present the coexistence of self-excited attractors and hidden attractors such that hidden attractors surround the self-excited attractors. The first part of the approach consists of the generation of self-excited attractors based on pairs of equilibria with heteroclinic orbits. Then, additional equilibria are added to the system to obtain a bistable system with a second self-excited attractor with the same characteristics. It is conjectured that a necessary condition for the existence of the hidden attractor in this class of systems is the rupture of the trajectories that resemble heteroclinic orbits that join the two regions of space that surround the pairs of equilibria; these regions resemble equilibria when seen on a larger scale. With the appearance of a hidden attractor, the system presents a multistable behavior with hidden and selfexcited attractors.
\end{abstract}

\section{Introduction}

There are two classes of attractors according to [1], which are defined as follows: the first class is given by those classical attractors excited from unstable equilibria called self-excited attractors whose basin of attraction intersects at least a neighborhood of an equilibrium point [2], and they are not difficult to find via numerical methods, and the second class is called hidden attractors whose basin of attraction does not contain neighborhoods of equilibria. The localization of this last class represents a more difficult task which has led to interesting approaches as the analytical-numerical algorithm suggested in [1] for the localization of hidden attractors of Chua's circuit.
Definition 1 (see [2])."An attractor is called a self-excited attractor if its basin of attraction intersects with any open neighborhood of an unstable fixed point. Otherwise, it is called a hidden attractor."

A hidden attractor is commonly observed in systems without equilibria or systems with a stable equilibrium point. Therefore, these classes of systems could serve as a starting point in the search for hidden attractors. However, according to the definition, a hidden attractor could be found in a system with any type and number of equilibria as well as any kind of attractors. Multistability is usually related to the existence of more than one attractor. Different scenarios of multistability are reported in [3]. 
Arnold Sommerfeld worked with one of the first dynamical systems with oscillating behavior but no equilibria [4]. In 1994, a conservative system without equilibria that presents a chaotic flow was reported in [5]. This system, known as Sprott case A, presents two quadratic nonlinearities, and it is a particular case of the Nose-Hoover system [6]. After this work, several three-dimensional systems without equilibria with chaotic attractors have been reported, like the one in [7] with two quadratic nonlinearities based on the Sprott system case $D$, the one in [8] with three quadratic nonlinearities, or the piecewise-linear system reported in [9]. In [10], three methods are used to produce seventeen three-dimensional systems without equilibria with chaotic flows, which present only quadratic nonlinearities.

Four-dimensional systems without equilibria with chaotic or hyperchaotic attractors have also been reported. For instance, systems with quadratic and cubic nonlinearities with hyperchaotic attractors are reported in $[11,12]$. The first piecewise-linear system without equilibria that exhibits a hyperchaotic attractor is reported in [13]. It is the result of the approximation made to the quadratic nonlinearities of an extended diffusionless Lorenz system. In [14], a fourdimensional system without equilibria with chaotic multiwing butterfly attractors is presented.

Since the double-scroll attractor in Chua's circuit, there exists an interest to generate double-scroll and multiscroll attractors. In circuits based on Chua's circuit, the implementation of piecewise-linear resistors with multiple segments is not an easy task due to their irregular breakpoints and slopes. Some approaches for self-excited scroll attractors have been reported in [15-19]. Recently, in [20], an approach for the generation of multiscroll hidden attractors with any number of scrolls in a system without equilibria was introduced. In [21], two systems with multiscroll hidden attractors are constructed by introducing nonlinear functions into Sprott system case A. In [22], a no-equilibrium system with a multiscroll hidden chaotic sea is introduced. In [23], a memristive system with chaotic attractors is presented. The multiscroll hidden attractors and multiwing hidden attractors exhibited by the system are sensitive to the transient simulation.

In [24], the widening of the basins of attraction of a class of piecewise-linear systems is studied. Also, a system with a double-scroll hidden attractor along with two double-scroll self-excited attractors is introduced. Based on this result, it is natural to think about the possibility of generating hidden attractors via multistable systems with double-scroll selfexcited attractors.

An approach that allows the generation of hidden attractors based on a kind of piecewise-linear (PWL) system is studied in this work. The study reveals a relationship between the emergence of a hidden attractor and the existence of trajectories that, when are seen on a larger scale, resemble heteroclinic orbits joining the self-excited attractors.

The study performed in this work suggests that some classes of systems with a multistable behavior could be designed geometrically to exhibit hidden and self-excited attractors. Chaotic scroll attractors have been widely studied and have been found useful in the design of pseudorandom number generation [25]. It has been demonstrated that the number of scrolls on some classes of systems affects the properties of the generated sequences determining if they fulfill the statistical test of the NIST and affecting the stream ciphering of images [26]. Some chaotic systems can be restored by reconstructing the attractor, which is not desirable in an encryption algorithm since it would reduce the security [27]. In a hidden scroll attractor, the restoration of the system is harder [27]. Thus, the class of systems discussed in this work could lead to the development of new cryptographic algorithms with more complex multistable systems with self-excited and hidden scroll attractors.

The structure of the article is as follows: In Section 2, a class of piecewise-linear systems with double-scroll selfexcited chaotic attractors is introduced. In Section 3, additional equilibria are considered to generate two self-excited attractors. In Section 4, the transitory behavior of the trajectories surrounding the self-excited attractors of the system is studied. In Section 5, the relation between the emergence of a hidden attractor and the existence of trajectories that, when are seen on a larger scale, resemble heteroclinic orbits joining the self-excited attractors is discussed. Finally, conclusions are given in Section 6 .

\section{Heteroclinic Chaos}

To introduce the approach, let us first consider a partition $P$ of the metric space $X \subset \mathbb{R}^{3}$, endowed with the Euclidean metric $d$. Let $P=\left\{P_{1}, \ldots, P_{\eta}\right\}(\eta>1)$ be a finite partition of $X$, that is, $X=\cup_{1 \leq i \leq \eta} P_{i}$, and $P_{i} \cap P_{j}=\varnothing$ for $i \neq j$. Each element of the set $P$ is called an atom and each atom contains a saddle equilibrium point. Due to these atoms, $P_{i}$ have a saddle equilibrium point, then within each atom there is a stable manifold and also an unstable manifold. These stable manifolds $W^{s}$ and unstable manifolds $W^{u}$ are necessary for the mechanism of expansion and contraction present in chaotic dynamics.

Let $T: X \longrightarrow X$, with $X \subset \mathbb{R}^{3}$, be a piecewise-linear dynamical system whose dynamics is given by a family of subsystems of the form

$$
\dot{x}=A \mathbf{x}+f(\mathbf{x}) B,
$$

where $\mathbf{x}=\left(x_{1}, x_{2}, x_{3}\right)^{T} \in \mathbb{R}^{3}$ is the state vector, and $A=$ $\left\{\alpha_{i j}\right\} \in \mathbb{R}^{3 \times 3}$ is a linear operator, $B=\left(\beta_{1}, \beta_{2}, \beta_{3}\right)^{T}$ is a constant vector, and $f$ is a functional. The vector $f(\mathbf{x}) B$ is a constant vector in each atom $P_{i}$ such that the equilibria are given by $\mathbf{x}_{\mathrm{eq}_{i}}^{*}=\left(x_{1_{\mathrm{eq}_{i}}^{*}}^{*}, x_{2_{\mathrm{eq}_{i}}^{*}}, x_{3_{\mathrm{eq}_{i}}^{*}}^{*}\right)^{T}=-f(\mathbf{x}) A^{-1} B \in P_{i}$, with $i=1, \ldots, \eta$.

Oscillations of the flow around the equilibria $\mathbf{x}_{\mathrm{eq}_{i}}^{*}$ are desired. Let us assign a negative real eigenvalue $\lambda_{1}=c$ to the complexification of the operator $A\left(A_{\mathbf{C}}\right)$ with the corresponding eigenvector $v_{1}$, and a pair of complex conjugate eigenvalues with positive real part $\lambda_{2}=a+i b$ and $\lambda_{3}=a-i b$ with the corresponding eigenvectors $v_{2}$ and $v_{3}$. Additionally, we restrict $b / a \geq 10$. Thus the stable and unstable manifolds are given by $W_{\mathbf{x}_{\mathrm{eq}_{i}}^{*}}^{s}=\left\{\mathbf{x}+\mathbf{x}_{\mathrm{eq}_{i}}^{*}: \mathbf{x} \in \operatorname{span}\left\{v_{1}\right\}\right\}$ and $W_{\mathbf{x}_{\mathrm{eq}_{i}}^{*}}^{u}=$ 
$\left\{\mathbf{x}+\mathbf{x}_{\mathrm{eq}_{i}}^{*}: \mathbf{x} \in \operatorname{span}\left\{v_{2}, v_{3}\right\}\right\}$, where $v_{1}, v_{2}$ and $v_{3}$ are given as follows:

$$
\begin{aligned}
& v_{1}=\left(\begin{array}{l}
1 \\
0 \\
1 \\
\frac{1}{2}
\end{array}\right), \\
& v_{2}=\left(\begin{array}{c}
0 \\
-1 \\
0
\end{array}\right), \\
& v_{3}=\left(\begin{array}{c}
-1 \\
0 \\
1
\end{array}\right) .
\end{aligned}
$$

The matrix of the linear operator $A$ is defined as follows:

$$
A=\left(\begin{array}{ccc}
\frac{a}{3}+\frac{2 c}{3} & b & \frac{2 c}{3}-\frac{2 a}{3} \\
-\frac{b}{3} & a & \frac{2 b}{3} \\
\frac{c}{3}-\frac{a}{3} & -b & \frac{2 a}{3}+\frac{c}{3}
\end{array}\right) .
$$

In this work, we denote the local stable and unstable manifolds of an equilibrium point $\mathbf{x}_{\mathrm{eq}}^{*}$ as $W_{\mathbf{x}_{\mathrm{eq}}^{*}}^{s}$ and $W_{\mathbf{x}_{\mathrm{eq}}^{*}}^{u}$ respectively, and they are responsible for connecting the equilibria of a dynamical system. Recall that a heteroclinic orbit is a path that joins two equilibrium points in the phase space. Similarly, a homoclinic orbit is a path that starts and ends at the same equilibrium point.

We also denoted the closure of a set $P_{i}$ as $\operatorname{cl}\left(P_{i}\right)$. Thus, for each pair of atoms $P_{i}$ and $P_{j}, i \neq j$, if $\operatorname{cl}\left(P_{i}\right) \cap \operatorname{cl}\left(P_{j}\right) \neq \varnothing$, then these atoms are adjacent and the switching surface between them is given by the intersection, i.e., $S W_{i j}=\mathrm{cl}\left(P_{i}\right) \cap \mathrm{cl}\left(P_{j}\right)$.

Each $\mathrm{SW}_{i j}$ has associated an equation of the form $\widehat{A} x_{1}+\widehat{B} x_{2}+\widehat{C} x_{3}+D=\mathbf{N}_{12} \cdot \mathbf{x}^{T}+D=0$, with $\widehat{A}>0$ where $\mathbf{N}_{12}=(\widehat{A}, \widehat{B}, \widehat{C})$ is the normal vector. Then the atoms $P_{i}, i=$ 1,2 are defined as follows:

$$
\begin{aligned}
P_{1} & =\left\{\mathbf{x} \in \mathbb{R}^{3}: x_{3}>0, \mathbf{N}_{12} \cdot \mathbf{x}^{T} \leq-D\right\} \\
& \cup\left\{\mathbf{x} \in \mathbb{R}^{3}: x_{3} \leq 0, \mathbf{N}_{12} \cdot \mathbf{x}^{T}<-D\right\}, \\
P_{2}= & \left\{\mathbf{x} \in \mathbb{R}^{3}: x_{3}>0, \mathbf{N}_{12} \cdot \mathbf{x}^{T}>-D\right\} \\
\cup & \left\{\mathbf{x} \in \mathbb{R}^{3}: x_{3} \leq 0, \mathbf{N}_{12} \cdot \mathbf{x}^{T} \geq-D\right\} .
\end{aligned}
$$

Remark 1. The divergence of the PWL system (1) considering the linear operator $A$ given by (3) is $\nabla=2 a+c$, so the system is dissipative in each atom of the partition $P$ if $2 a<|c|$.

With the atoms of a $P$ partition containing a saddle equilibrium point in each of them as defined above, it is possible to generate heteroclinic orbits. To generate a heteroclinic orbit, at least two equilibria are required. Therefore, consider a partition with two atoms $P=\left\{P_{1}, P_{2}\right\}$, the constant vector $B \in \mathbb{R}^{3}$ is defined as follows:

$$
B=\left(\begin{array}{c}
-\frac{a}{3}-\frac{2 c}{3} \\
\frac{b}{3} \\
\frac{a}{3}-\frac{c}{3}
\end{array}\right),
$$

and the functional $f$ is given by

$$
f(\mathbf{x})= \begin{cases}-\alpha, & \mathbf{x} \in P_{1}, \\ \alpha, & \mathbf{x} \in P_{2},\end{cases}
$$

with $\alpha>0$. So the equilibria are at $\mathbf{x}_{\mathrm{eq}}^{*}=(-\alpha, 0,0)^{T} \in P_{1}$ and $\mathbf{x}_{\mathrm{eq}_{2}}^{*}=(\alpha, 0,0)^{T} \in P_{2}$, and the stable and the unstable manifolds are given by

$$
\begin{aligned}
W_{\mathrm{x}_{\mathrm{eq}}^{*}}^{s} & =\left\{\mathbf{x} \in \mathbb{R}^{3}: \mid x_{1}+\alpha=2 x_{3}, x_{2}=0\right\}, \\
W_{\mathrm{x}_{\mathrm{eq}}}^{u} & =\left\{\mathbf{x} \in \mathbb{R}^{3}: \mid x_{1}+x_{3}=-\alpha\right\}, \\
W_{\mathrm{x}_{\mathrm{eq}}}^{s} & =\left\{\mathbf{x} \in \mathbb{R}^{3}: \mid x_{1}-\alpha=2 x_{3}, x_{2}=0\right\}, \\
W_{\mathrm{x}_{\mathrm{eq}}}^{u} & =\left\{\mathbf{x} \in \mathbb{R}^{3}: \mid x_{1}+x_{3}=\alpha\right\} .
\end{aligned}
$$

Proposition 1 (see $[28,29])$. "The hyperbolic system given by (1), (3), (5), and (6) generates a pair of heteroclinic orbits if the switching surface between the atoms $P_{1}$ and $P_{2}$ is given by the plane $S W_{12}=\left\{\mathbf{x} \in \mathbb{R}^{3}: 2 x_{1}-x_{3}=0\right\}$.”

The points where the stable and unstable manifolds intersect at $S W$ are given by

$$
\begin{aligned}
& \mathbf{x}_{i n_{1}}=\operatorname{cl}\left(W_{\mathbf{x}_{\mathrm{eq}_{1}}^{*}}^{s}\right) \cap \mathrm{cl}\left(W_{\mathbf{x}_{\mathrm{eq}_{2}}^{*}}^{u}\right)=\left(\frac{\alpha}{3}, 0, \frac{2 \alpha}{3}\right)^{T}, \\
& \mathbf{x}_{\text {in }_{2}}=\operatorname{cl}\left(W_{\mathrm{x}_{\mathrm{eq}_{2}}^{*}}^{s}\right) \cap \mathrm{cl}\left(W_{\mathrm{x}_{\mathrm{eq}_{1}}^{*}}^{u}\right)=\left(-\frac{\alpha}{3}, 0,-\frac{2 \alpha}{3}\right)^{T} .
\end{aligned}
$$

These points $\mathbf{x}_{\mathrm{in}_{1}}$ and $\mathbf{x}_{\mathrm{in}_{2}}$ belong to $S W_{12}$ and $\mathbf{x}_{\mathrm{in}_{1}} \in P_{1}$ and $\mathbf{x}_{\mathrm{in}_{2}}=P_{2}$. Because these points $\mathbf{x}_{\mathrm{in}_{1}}$ and $\mathbf{x}_{\mathrm{in}}$ belong to the stable manifolds $W_{\mathbf{x}_{\mathrm{eq}}^{*}}^{s}$ and $W_{\mathbf{x}_{\mathrm{eq}}^{*}}^{s}$, respectively, they are points whose trajectories remain in atoms $P_{1}$ and $P_{2}$, respectively. Thus, the heteroclinic orbits are defined as follows:

$$
\begin{aligned}
& \mathrm{HO}_{1}=\left\{\mathbf{x} \in \varphi\left(\mathbf{x}_{i \eta_{1}}, t\right): t \in(-\infty, \infty)\right\} \\
& \mathrm{HO}_{2}=\left\{\mathbf{x} \in \varphi\left(\mathbf{x}_{i \eta_{2}}, t\right): t \in(-\infty, \infty)\right\} .
\end{aligned}
$$


For the system given by (1), (3), (5), and (6), it is possible to find several points $\mathbf{x}_{0} \in \mathrm{HO}_{i}$ such that $\left|\mathbf{x}_{\mathrm{eq}_{i}}-\mathbf{x}_{0}\right|<\varepsilon$ with $\varepsilon$ arbitrarily small and $i=1,2$. Thus, one can find initial conditions for the simulation of the heteroclinic orbits as close to the equilibria as desired. One example of the initial condition formula for $P_{1}$ is as follows:

$$
\mathbf{x}_{0}^{1}=\left(\begin{array}{c}
\frac{2}{3} \alpha e^{-(2 k a \pi / b)}-\alpha \\
0 \\
-\frac{2}{3} \alpha e^{-(2 k a \pi / b)}
\end{array}\right)
$$

and for $P_{2}$

$$
\mathbf{x}_{0}^{2}=\left(\begin{array}{c}
-\frac{2}{3} \alpha e^{-(2 k a \pi / b)}+\alpha \\
0 \\
\frac{2}{3} \alpha e^{-(2 k a \pi / b)}
\end{array}\right)
$$

with $k \in \mathbb{Z}^{+}$.

Example 1. Consider the system (1), (3), (5), and (6) with $\mathrm{SW}_{12}=\left\{\mathbf{x} \in \mathbb{R}^{3}: 2 x_{1}-x_{3}=0\right\}$ and the parameters $a=0.2, b=5, c=-3, \alpha=1$.

The above-defined system fulfills Proposition 1, so it presents a heteroclinic orbit. From (10) and (11), two initial conditions,

$$
\begin{aligned}
& \mathbf{x}_{01}=(-0.9999976751050959,0,-2.3248949041393315 e-6)^{T}, \\
& \mathbf{x}_{02}=(0.9999976751050959,0,2.3248949041393315 e-6)^{T},
\end{aligned}
$$

are chosen with $k=50$ to simulate the two heteroclinic orbits shown in Figure 1(a). A double-scroll attractor with heteroclinic chaos is generated, and it is shown in Figure 1(a) for the initial condition $\mathbf{x}_{0}=(0,0,0)^{T}$.

The unstable manifolds $W_{\mathbf{x}^{*}}^{u}=\left\{\mathbf{x} \in \mathbb{R}^{3}: x_{1}+x_{3}+1=\right.$ $0\}$ and $W_{\mathbf{x}^{*}}^{u}=\left\{\mathbf{x} \in \mathbb{R}^{3}: x_{1}+x_{3}-1=0\right\}$ and the stable manifolds $W_{\mathbf{x}^{*}}^{s}=\left\{\mathbf{x} \in \mathbb{R}^{3}:\left(x_{1}+1\right) / 2=x_{3} ; x_{2}=0\right\}$ and $W_{\mathbf{x}_{\text {eq }}^{*}}^{s}=\left\{\mathbf{x} \in \mathbb{R}^{3^{\text {eq }_{1}}:}\left(x_{1}-1\right) / 2=x_{3} ; x_{2}=0\right\}$. The intersection points are given by $\operatorname{cl}\left(W_{\mathbf{x}^{*}}^{s}\right) \cap \mathrm{cl}\left(W_{\mathbf{x}^{*}}^{u}\right)=(-(1$ /3), $0,-(2 / 3))^{T}, \operatorname{cl}\left(W_{\mathbf{x}_{\mathrm{eq}_{1}}^{*}}^{s}\right) \cap \operatorname{cl}\left(W_{\mathbf{x}_{\mathrm{eq}_{2}}^{*}}^{u}\right)=((1 / 3), 0,(2 / 3))^{T}$.

Proposition 2 (see [29]). "If the partition $P$ contains more than two atoms $\left\{P_{1}, P_{2}, \ldots, P_{k}\right\}$, with $2<k \in \mathbb{Z}^{+}$, and each atom is a hyperbolic set defined as above. Furthermore, the atoms by pairs $P_{i}$ and $P_{i+1}$ fulfill Proposition 1. Then, the system generates $2(k-1)$ heteroclinic orbits."
Proof. A direct consequence of Proposition 1.

\section{Emergence of Multiscroll Attractors through Multiple Heteroclinic Orbits}

According to the Proposition 2, it is possible to generate multiscroll attractors based on multiple heteroclinic orbits. So in this Section, we consider more than two hyperbolic sets in the partition with the aim of studying the existence of heterocyclic cycles and the attractors exhibited by the system when varying the location of the equilibria.

Consider the partition $P=\left\{P_{1}, P_{2}, P_{3}, P_{4}\right\}$ along with the piecewise-linear dynamical system (1), with $A$ and $B$ given by (3) and (5), respectively. Thus the function $f(\mathbf{x})$ is defined in the four atoms as follows:

$$
f(\mathbf{x})= \begin{cases}-\alpha-\gamma, & \mathbf{x} \in P_{1}, \\ \alpha-\gamma, & \mathbf{x} \in P_{2}, \\ -\alpha+\gamma, & \mathbf{x} \in P_{3}, \\ \alpha+\gamma, & \mathbf{x} \in P_{4},\end{cases}
$$

where $\alpha, \gamma>0$. The equilibria are at

$$
\begin{aligned}
& \mathbf{x}_{\mathrm{eq}_{1}}^{*}=\left[\begin{array}{c}
-(\gamma+\alpha) \\
0 \\
0
\end{array}\right], \\
& \mathbf{x}_{\mathrm{eq}_{2}}^{*}=\left[\begin{array}{c}
-(\gamma-\alpha) \\
0 \\
0
\end{array}\right], \\
& \mathbf{x}_{\mathrm{eq}_{3}}^{*}=\left[\begin{array}{c}
(\gamma-\alpha) \\
0 \\
0
\end{array}\right], \\
& \mathbf{x}_{\mathrm{eq}_{4}}^{*}=\left[\begin{array}{c}
(\gamma+\alpha) \\
0 \\
0
\end{array}\right],
\end{aligned}
$$

so $\mathbf{x}_{\mathrm{eq}_{1}}^{*} \in P_{1}, \mathbf{x}_{\mathrm{eq}_{2}}^{*} \in P_{2}, \mathbf{x}_{\mathrm{eq}_{3}}^{*} \in P_{3}$ and $\mathbf{x}_{\mathrm{eq}_{4}}^{*} \in P_{4}$. The location of the equilibria according to the parameters $0<\alpha$ and $0<\gamma$ is as follows:

(i) The equilibria are on the $x_{1}$ axis and for $\alpha=\gamma$ the system only have three equilibria. Otherwise, it has four equilibria.

(ii) For $\alpha<\gamma$ the distance of the equilibria $\mathbf{x}_{\mathrm{eq}_{1}}^{*}$ and $\mathbf{x}_{\mathrm{eq}_{4}}^{*}$ to the origin $O=(0,0,0)^{T}$ are the same $d\left(\mathbf{x}_{\mathrm{eq}_{1}}^{*}, O\right) \stackrel{\mathrm{eq}_{4}}{=}$ $d\left(\mathbf{x}_{\mathrm{eq}_{4}}^{*}, O\right)$ and also for $d\left(\mathbf{x}_{\mathrm{eq}_{2}}^{*}, O\right)=d\left(\mathbf{x}_{\mathrm{eq}_{3}}^{*}, O\right)$.

(iii) For $\gamma=2 \alpha$, all equilibria are at the same distance $d\left(\mathbf{x}_{\mathrm{eq}_{1}}^{*}, \mathbf{x}_{\mathrm{eq}_{2}}^{*}\right)=d\left(\mathbf{x}_{\mathrm{eq}_{2}}^{*}, \mathbf{x}_{\mathrm{eq}_{3}}^{*}\right)=d\left(\mathbf{x}_{\mathrm{eq}_{3}}^{*}, \mathbf{x}_{\mathrm{eq}_{4}}^{*}\right)=2 \alpha$.

(iv) The other case is when $\gamma \neq 2 \alpha$, and $d\left(\mathbf{x}_{\mathrm{eq}_{1}}^{*}, \mathbf{x}_{\mathrm{eq}_{2}}^{*}\right)$ $=d\left(\mathbf{x}_{\mathrm{eq}_{3}}^{*}, \mathbf{x}_{\mathrm{eq}_{4}}^{*}\right)=2 \alpha$, but $d\left(\mathbf{x}_{\mathrm{eq}_{2}}^{*}, \mathbf{x}_{\mathrm{eq}_{3}}^{*}\right) \neq 2 \alpha$.

In this section, we are especially interested in the case of $\gamma \neq 2 \alpha$ such that $\gamma>\alpha$ with switching surfaces given by 


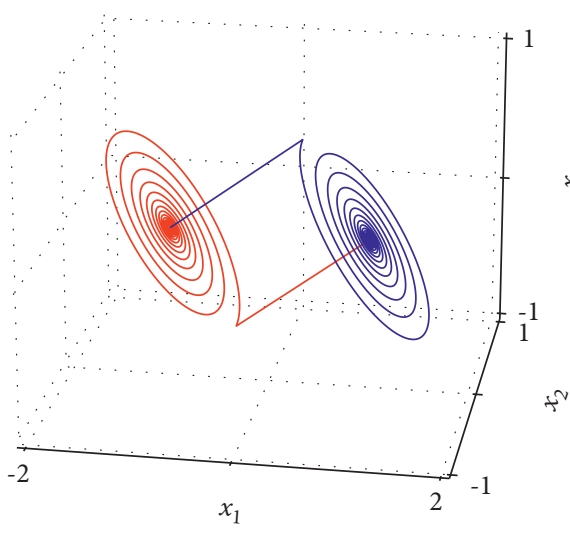

(a)

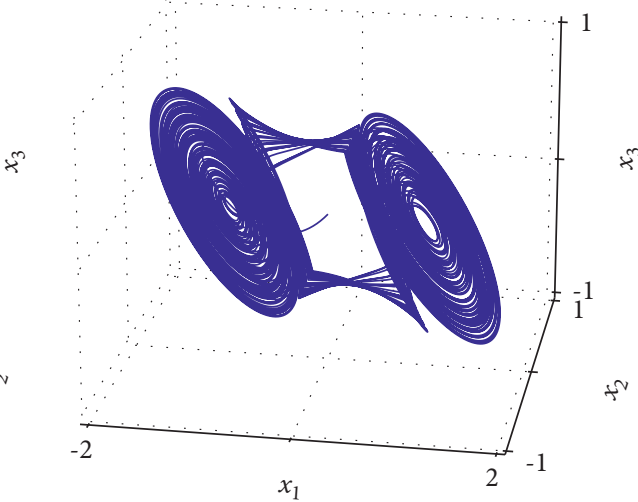

(b)

FiguRE 1: In (a) the heteroclinic loop of the system (1), (3), (5), and (6) with the switching surface $\left\{x \in \mathbb{R}^{3}: 2 x_{1}-x_{3}=0\right\}$, the parameters $a=0.2, b=5, c=-3, \alpha=1$, and the initial conditions $\mathbf{x}_{01}=(-0.9999976751050959,0,-2.3248949041393315 e-6)^{T} \quad($ red $)$ and $\mathbf{x}_{02}=(0.9999976751050959,0,2.3248949041393315 e-6)^{T}$ (blue), and in (b) a double-scroll attractor that emerges from a heteroclinic orbit using the following initial condition $\mathbf{x}_{0}=(0,0,0)^{T}$ and the same parameters.

$$
\begin{aligned}
& S W_{12}=\operatorname{cl}\left(P_{1}\right) \cap \operatorname{cl}\left(P_{2}\right)=\left\{\mathbf{x} \in \mathbb{R}^{3}: 2 x_{1}-x_{3}=-2 \gamma\right\}, \\
& S W_{23}=\operatorname{cl}\left(P_{2}\right) \cap \operatorname{cl}\left(P_{3}\right)=\left\{\mathbf{x} \in \mathbb{R}^{3}: 2 x_{1}-x_{3}=0\right\}, \\
& S W_{34}=\operatorname{cl}\left(P_{3}\right) \cap \operatorname{cl}\left(P_{4}\right)=\left\{\mathbf{x} \in \mathbb{R}^{3}: 2 x_{1}-x_{3}=2 \gamma\right\},
\end{aligned}
$$

which fulfill that

$$
\begin{aligned}
& \operatorname{SW}_{i(i+1)} \cap\left\{\mathbf{x} \in \mathbb{R}^{3}: x_{3}>0\right\} \in P_{i}, \\
& \operatorname{SW}_{i(i+1)} \cap\left\{\mathbf{x} \in \mathbb{R}^{3}: x_{3} \leq 0\right\} \in P_{i+1} .
\end{aligned}
$$

This way of defining the switching surfaces provokes that the intersections between them and the stable manifolds contain a point, and the intersections between them and the unstable manifolds are the empty set, i.e., $W_{\mathbf{x}_{\text {eq }_{1}}^{*}}^{u} \cap \mathrm{SW}_{12}=\varnothing$ and $W_{\mathbf{x}^{*}}^{s} \cap S W_{12} \neq \varnothing$.

Let us define two points, $p b=W_{\mathbf{x}_{e q}^{*}}^{s} \cap S W_{12}$ and $p a=$ $W_{\mathbf{x}_{\mathrm{eq}_{3}}^{*}}^{s} \cap S W_{23}$ as shown in Figure 2. Then $p a$ and $p b$ are given as follows:

$$
\begin{aligned}
& p a=\left(\begin{array}{c}
-\frac{(\gamma-\alpha)}{3} \\
0 \\
2(\gamma-\alpha) \\
-\frac{\alpha}{3}
\end{array}\right), \\
& p b=\left(\begin{array}{c}
\frac{\alpha}{3}-\gamma \\
0 \\
\frac{2 \alpha}{3}
\end{array}\right) .
\end{aligned}
$$

The set $\operatorname{cl}\left(W_{\mathbf{X}_{\text {eq }}^{*}}^{u}\right) \cap S W_{12}$ can be written as follows:

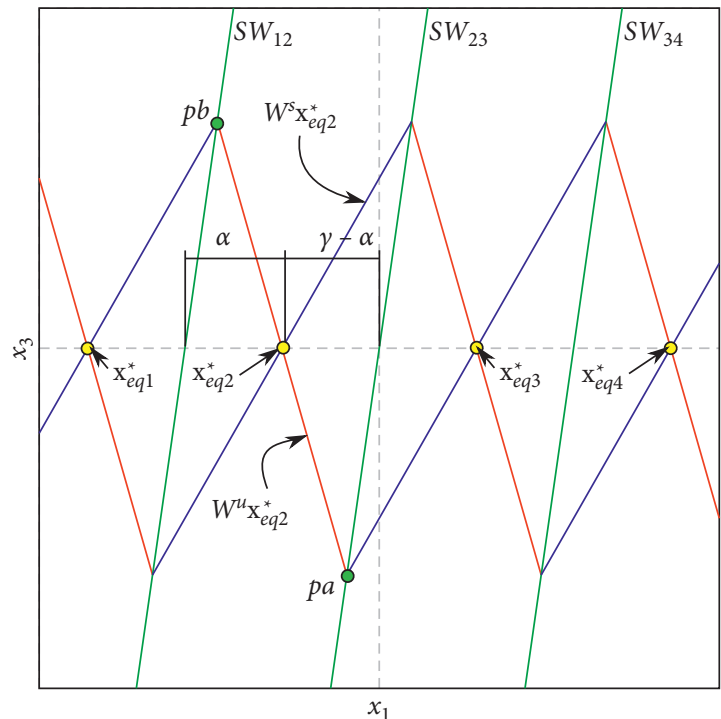

Figure 2: Projection of the stable and unstable manifolds and switching planes onto the $x_{1}-x_{3}$ plane. The diagram shows the location of the unstable manifold marked with red lines, the stable manifold marked with blue lines, and switching planes marked with green lines.

$$
\left\{\mathbf{x} \in \mathbb{R}^{3}: \mathbf{x}=(0, \mathcal{\varepsilon}, 0)^{T}+p b, \varepsilon \in \mathbb{R}\right\},
$$

and the set $\operatorname{cl}\left(W_{\mathbf{x}_{\mathrm{q}_{2}}^{*}}^{u}\right) \cap S W_{23}$ can be written as follows:

$$
\left\{\mathbf{x} \in \mathbb{R}^{3}: \mathbf{x}=(0, \mathcal{\varepsilon}, 0)^{T}+p a, \varepsilon \in \mathbb{R}\right\} .
$$

Consider the transformation $\mathbf{z}^{(2)}=Q^{-1}\left(\mathbf{x}-\mathbf{x}_{\text {eq }}^{*}\right)$, since $Q^{-1}(0, \varepsilon, 0)^{T}=(0,-\mathcal{E}, 0)^{T}$, so $\quad \mathbf{z}^{(2)}=(0, \varepsilon, 0)^{T} \quad+Q^{-1}(p b$ $\left.-\mathbf{x}^{*} \mathrm{q}_{2}\right)$, where $Q^{-1}\left(p b-\mathbf{x}_{\mathrm{eq}_{2}}^{*}\right)$ is a point on the plane $z_{2}^{(2)^{2}}-z_{3}^{(2)}$. And $\mathbf{z}^{(2)}=(0, \varepsilon, 0)^{T}+Q^{-1}\left(p a-\mathbf{x}_{\mathrm{eq}_{2}}^{*}\right)$, where $\mathrm{Q}^{-1}\left(p a-\mathbf{x}_{\mathrm{eq}_{2}}^{*}\right)$ is also a point on the plane $z_{2}^{(2)}-z_{3}^{(2)}$. Then, the set (18) in $\mathbf{z}^{(2)}$ coordinates is given by 


$$
\left\{\mathbf{z}^{(2)} \in \mathbb{R}^{3}: \mathbf{z}^{(2)}=\left(0, \varepsilon, \frac{2 \alpha}{3}\right)^{T}, \varepsilon \in \mathbb{R}\right\},
$$

and the set (19) in $\mathbf{z}^{(2)}$ coordinates is given by

$$
\left\{\mathbf{z}^{(2)} \in \mathbb{R}^{3}: \mathbf{z}^{(2)}=\left(0, \varepsilon, \frac{2(\alpha-\gamma)}{3}\right)^{T}, \varepsilon \in \mathbb{R}\right\} .
$$

Thus, the sets (20) and (21) are orthogonal lines to the $z_{3}^{(2)}$ axis. The points $p a$ and $p b$ in $\mathbf{z}^{(2)}$ coordinates will be denoted as follows:

$$
\begin{aligned}
& p a_{z}=\left(\begin{array}{c}
0 \\
0 \\
\frac{2(\alpha-\gamma)}{3}
\end{array}\right), \\
& p b_{z}=\left(\begin{array}{c}
0 \\
0 \\
\frac{2 \alpha}{3}
\end{array}\right) .
\end{aligned}
$$

With the uncoupled system in $\mathbf{z}^{(2)}$ coordinates, we can analyze the flow on the plane $z_{2}^{(2)}-z_{3}^{(2)}$ close to $\mathbf{z}_{\mathrm{eq}_{2}}^{*(2)}$.

$$
\begin{aligned}
\dot{z}_{2}^{(2)} & =a z_{2}^{(2)}-b z_{3}^{(2)}, \\
\dot{z}_{3} & =b z_{2}^{(2)}+a z_{3}^{(2)}, \\
r \dot{r} & =z_{2}^{(2)} \dot{z}_{2}^{(2)}+z_{3}^{(2)} \dot{z}_{3}^{(2)}=a r^{2}, \\
\dot{r} & =a r \\
r & =r_{0} e^{a t} .
\end{aligned}
$$

It follows from (22) that if $\alpha=\gamma-\alpha$, then the points $p a_{z}$ and $p b_{z}$ are at the same distance from $\mathbf{z}_{\mathrm{eq}_{2}}^{*(2)}=(0,0,0)^{T^{2}}$. Thus, from (26), it follows that the trajectories with initial conditions $p a_{z}$ and $p b_{z}$ remain in $P_{2}$ for all $t<0$.

Our case study is $\gamma-\alpha \neq \alpha$, such that $\gamma>\alpha$. Let us consider the case $\gamma-\alpha>\alpha$, and it can be seen from (22) that $p b_{z}$ is closer to $\mathbf{z}_{\mathrm{eq}_{2}}^{(2)}$ than $p a_{z}$, this is, $d\left(p b_{z}, \mathbf{z}_{\mathrm{eq}_{2}}^{(2)}\right)$ $<d\left(p a_{z}, \mathbf{z}_{\mathrm{eq}_{2}}^{(2)}\right)$. Then, if $\gamma$ is sufficiently big with respect to $\alpha$, the trajectory with the initial condition $p a_{z}$ will eventually reach the set given by (20) for $t<0$; i.e., the trajectory of the initial condition $p a \in \mathrm{SW}_{23}$ reaches the switching plane $\mathrm{SW}_{12}$ and not the equilibrium point $\mathbf{x}_{\mathrm{eq}_{2}^{*}}^{*}$. This means that in $\mathbf{x}$ coordinates, the heteroclinic orbit from $\mathbf{x}_{\mathrm{eq}_{2}}^{*}$ to $\mathbf{x}_{\mathrm{eq}_{3}}^{*}$ does not exist. Similarly, when $p b_{z}$ is further than $p a_{z}$ from $\mathbf{z}_{\mathrm{eq}_{2}}^{*(2)}$, this is, $d\left(p a_{z}, \mathbf{z}_{\mathrm{eq}}^{(2)}\right)<d\left(p b_{z}, \mathbf{z}_{\mathrm{eq}_{2}}^{(2)}\right)$, for $\gamma$ sufficiently small, the trajectory with the initial condition $p b_{z}$ will eventually reach the set given by (21) for $t<0$, i.e., the trajectory of the initial condition $p b \in \mathrm{SW}_{12}$ reaches the switching plane $\mathrm{SW}_{23}$ and not the equilibrium point $\mathbf{x}_{\mathrm{eq}_{2}}^{*}$. This means that in $\mathbf{x}$ coordinates, the heteroclinic orbit from $\mathbf{x}_{\mathrm{eq}_{2}}^{*}$ to $\mathbf{x}_{\mathrm{eq}_{1}}^{*}$ does not exist.

The next proposition warranty the existence of heteroclinic orbits when $\gamma$ belongs to an interval of real numbers where the case $\gamma-\alpha \neq \alpha$ is considered, such that $\gamma>\alpha$.

Proposition 3. The hyperbolic system given by (1), (3), (5), and (13) with the switching surfaces given in (15) generates six heteroclinic orbits if

$$
\frac{\alpha\left(e^{-a \tau} \cos (b \tau)-1\right)}{e^{-a \tau} \cos (b \tau)}>\gamma>\alpha\left(1-e^{-a \tau} \cos (b \tau)\right),
$$

where

$$
\tau=\frac{\arctan (b / a)+\pi / 2}{b} .
$$

Proof. To find the values of $\gamma$ for which these heteroclinic orbits exist, let us assume $p a$ is a point of the heteroclinic orbit joining $\mathbf{x}_{\mathrm{eq}_{2}}^{*}$ and $\mathbf{x}_{\mathrm{eq}_{3}}^{*}$, i.e.,

$$
\begin{aligned}
& \lim _{t \longrightarrow-\infty} \varphi(p a, t)=\mathbf{x}_{\mathrm{eq}_{2}}^{*}, \\
& \lim _{t \longrightarrow \infty} \varphi(p a, t)=\mathbf{x}_{\mathrm{eq}_{3}}^{*} .
\end{aligned}
$$

Because $p a \in W_{\mathbf{x}_{\mathrm{eq} 3}^{*}}^{s}$ then $\lim _{t \longrightarrow \infty} \varphi(p a, t)=\mathbf{x}_{\mathrm{eq}_{3}}^{*}$. For the other part of the heteroclinic orbit, we analyze the system in $\mathbf{z}^{(2)}$ coordinates, we have $p a_{z}, p b_{z}, \mathbf{z}_{\mathrm{eq}_{2}}^{*(2)}$, and the orbit is given by $\mathbf{z}^{(2)}(t)$. We assume that $\mathbf{z}^{(2)}(0)=p a_{z}$, so we want that $\mathbf{z}^{(2)}(t)$ remains in $P_{2}$ for all $t<0$. Thus, we need to find the first maximum in the component $z_{3}^{(2)}$ of the trajectory whose initial condition is $p a_{z}$ for $t<0$. According to (22), the third component of $p a_{z}$ and $p b_{z}$ are $(2(\alpha-\gamma) / 3)<0$ and $0<(2 \alpha / 3)$, respectively. This maximum gives us the intersection point between the trajectory $\mathbf{z}^{(2)}(t)$ and the axis $z_{3}^{(2)}$. Then we can compare the third component of the trajectory $\mathbf{z}^{(2)}(t)$ and the point $p b_{z}$, in terms of $\alpha$ and $\gamma$ to ensure that $\mathbf{z}^{(2)}(t)$ remains in $P_{2}$ for all $t<0$.

The trajectory $\mathbf{z}^{(2)}(t)$ for the initial condition $\mathbf{z}_{0}^{(2)}=\left(z_{10}^{(2)}, z_{20}^{(2)}, z_{30}^{(2)}\right)^{T}$ is as follows:

$$
\begin{aligned}
z_{1}^{(2)}(t) & =z_{10}^{(2)} e^{-c t}, \\
z_{2}^{(2)}(t) & =z_{20}^{(2)} e^{a t} \cos (b t)-z_{30}^{(2)} e^{a t} \sin (b t), \\
z_{3}^{(2)}(t) & =z_{20}^{(2)} e^{a t} \sin (b t)+z_{30}^{(2)} e^{a t} \cos (b t) .
\end{aligned}
$$

This set of equations is analyzed for $t<0$. The same analysis can be done for $0<t$ by using the following set of equations:

$$
\begin{aligned}
& z_{1}^{(2)}(t)=z_{10}^{(2)} e^{c t} \\
& z_{2}^{(2)}(t)=z_{20}^{(2)} e^{-a t} \cos (b t)+z_{30}^{(2)} e^{-a t} \sin (b t), \\
& z_{3}^{(2)}(t)=-z_{20}^{(2)} e^{-a t} \sin (b t)+z_{30}^{(2)} e^{-a t} \cos (b t) .
\end{aligned}
$$
$0<t$.

Since we are looking for the first maximum in $z_{3}^{(2)}(t)$ for Then from (33) with the initial condition $p a_{z}$ given in (22), 


$$
\begin{aligned}
& z_{3}^{(2)}(t)=\frac{2(\alpha-\gamma)}{3} e^{-a t} \cos (b t), \\
& \dot{z}_{3}^{(2)}(t)=-\frac{2(\alpha-\gamma)}{3} e^{-a t}(b \sin (b t)+a \cos (b t)), \\
& \dot{z}_{3}^{(2)}(t)=-\frac{2(\alpha-\gamma)}{3} e^{-a t}\left(\sqrt{a^{2}+b^{2}} \cos \left(b t-\arctan \left(\frac{b}{a}\right)\right)\right),
\end{aligned}
$$

to find the maximum, we equate to zero

$$
0=-\frac{2(\alpha-\gamma)}{3} e^{-a t}\left(\sqrt{a^{2}+b^{2}} \cos \left(b t-\arctan \left(\frac{b}{a}\right)\right)\right) .
$$

Thus, it turns out that

$$
\begin{aligned}
b t-\arctan \left(\frac{b}{a}\right) & =\frac{\pi}{2}+n \pi, \quad \text { with } n \in \mathbb{Z}, \\
t & =\frac{\arctan (b / a)}{b}+\frac{\pi}{2 b}+\frac{n \pi}{b}, \quad \text { with } n \in \mathbb{Z} .
\end{aligned}
$$

We will call $t_{\max }$ the time for the first maximum. Thus, it follows that

$$
t_{\max }=\frac{\arctan (b / a)+\pi / 2}{b}
$$

then from (34),

$$
z_{3}^{(2)}\left(t_{\max }\right)=\frac{2(\alpha-\gamma)}{3} e^{-a t_{\max }} \cos \left(b t_{\max }\right) .
$$

This maximum $z_{3}^{(2)}$ must be part of $P_{2}$, since $p b_{z}$ belongs to $P_{1}$ it follows from (22) that

$$
\begin{aligned}
& \frac{2 \alpha}{3}>\frac{2(\alpha-\gamma)}{3} e^{-a t_{\max }} \cos \left(b t_{\max }\right), \\
& \frac{\alpha\left(e^{-a t_{\max }} \cos \left(b t_{\max }\right)-1\right)}{e^{-a t_{\max }} \cos \left(b t_{\max }\right)}>\gamma .
\end{aligned}
$$

Now, let us assume $p b$ is a point of the heteroclinic orbit joining $\mathbf{x}_{\mathrm{eq}_{2}}^{*}$ and $\mathbf{x}_{\mathrm{eq}_{1}}^{*}$, i.e.,

$$
\begin{aligned}
& \lim _{t \longrightarrow-\infty} \varphi(p b, t)=\mathbf{x}_{\mathrm{eq}_{2}}^{*}, \\
& \lim _{t \longrightarrow \infty} \varphi(p b, t)=\mathbf{x}_{\mathrm{eq}_{1}}^{*} .
\end{aligned}
$$

Because $p b \in W_{\mathbf{x}_{\mathrm{eq}}^{*}}^{s}$ then $\lim _{t \rightarrow \infty} \varphi(p b, t)=\mathbf{x}_{\mathrm{eq}_{1}}^{*}$.

Following the same procedure described above but looking for a minimum, due to the third component of $p b_{z}$ is $0<(2 \alpha / 3)$. It is found that

$$
t_{\min }=\frac{\arctan (b / a)+\pi / 2}{b}
$$

Then from (33) and the point $p b_{z}$ given in (22),

$$
z_{3}^{(2)}\left(t_{\min }\right)=\frac{2 \alpha}{3} e^{-a t_{\min }} \cos \left(b t_{\min }\right) \text {. }
$$

This minimum $z_{3}^{(2)}$ must be part of $P_{2}$, since $p a_{z}$ belongs to $P_{3}$ it follows from (22) that

$$
\begin{aligned}
\frac{2(\alpha-\gamma)}{3} & <\frac{2 \alpha}{3} e^{-a t_{\min }} \cos \left(b t_{\min }\right), \\
\gamma & >\alpha\left(1-e^{-a t_{\min }} \cos \left(b t_{\min }\right)\right) .
\end{aligned}
$$

Then defining $\tau=t_{\max }=t_{\min }$

$$
\frac{\alpha\left(e^{-a \tau} \cos (b \tau)-1\right)}{e^{-a \tau} \cos (b \tau)}>\gamma>\alpha\left(1-e^{-a \tau} \cos (b \tau)\right) \text {. }
$$

The same conclusion applies to the point $\mathbf{x}_{\mathrm{eq}_{3}}^{*}$ due to the symmetry of the system. Finally, the heteroclinic orbit from $\mathbf{x}_{\mathrm{eq}_{1}}^{*}$ to $\mathbf{x}_{\mathrm{eq}_{2}}^{*}$ and the one from $\mathbf{x}_{\mathrm{eq}_{4}}^{*}$ to $\mathbf{x}_{\mathrm{eq}_{3}}^{*}$ are always present in the system as there are no more switching surfaces.

To illustrate the effect of the parameters $\gamma, \alpha, a$, and $b$ on the existence of heteroclinic orbits of the system given by (1), (3), (5), (13), and (15), we use Proposition 3 to determine the open interval of real values for $\gamma$ given by

$$
\Gamma=\left(\alpha\left(1-e^{-a \tau} \cos (b \tau)\right), \frac{\alpha\left(e^{-a \tau} \cos (b \tau)-1\right)}{e^{-a \tau} \cos (b \tau)}\right),
$$

with $\tau=(\arctan (b / a)+\pi / 2) / b$. So, six initial conditions were calculated as in (10) and (11) with $k=50$ for the parameters $a=0.2, b=5, c=-3$, and $\alpha=1$. Four cases of different values of $\gamma$ are analyzed. The first two correspond to $\gamma_{1,2} \in \Gamma$ and the last two correspond to $\gamma_{L, U} \notin \Gamma$ :.

(1) For this case $\gamma_{1} \in \Gamma$, with $\gamma_{1}=\alpha\left(1-e^{-a \tau} \cos (b \tau)\right)+0.00001$, so there exist six heteroclinic orbits, as shown in Figure 3(a).

(2) For $\gamma_{2}=\left(\left(\alpha\left(e^{-a \tau} \cos (b \tau) \quad-1\right) / e^{-a \tau} \cos (b \tau)\right)\right.$ $-0.00001) \in \Gamma$, in this case, there exist also six heteroclinic orbits as shown in Figure 3(b).

(3) In this case $\gamma_{L}=\alpha\left(1-e^{-a \tau} \cos (b \tau)\right) \notin \Gamma$, then there exist four heteroclinic orbits, as shown in Figure 3(c). The green orbit stating close to $\mathbf{x}_{\mathrm{eq}_{2}}^{*}$ cannot reach $\mathbf{x}_{\mathrm{eq}_{1}}^{*}$ and goes to $P_{3}$. In the same way, the yellow orbit starting close to $\mathbf{x}_{\mathrm{eq}_{3}}^{*}$ cannot reach $\mathbf{x}_{\mathrm{eq}_{4}}^{*}$ and goes to $P_{2}$. Then there is no heteroclinic orbits from $\mathbf{x}_{\mathrm{eq}_{2}}^{*}$ to $\mathbf{x}_{\mathrm{eq}_{1}}^{*}$ and from $\mathbf{x}_{\mathrm{eq}_{3}}^{*}$ to $\mathbf{x}_{\mathrm{eq}_{4}}^{*}$.

(4) For $\quad \gamma_{U}=\left(\alpha\left(e^{-a \tau} \cos (b \tau)-1\right) / e^{-a \tau} \cos (b \tau)\right) \notin \Gamma$, there also exist four heteroclinic orbits as shown in Figure $3(\mathrm{~d})$. The red orbit stating close to $\mathbf{x}_{\mathrm{eq}_{2}}^{*}$ cannot reach $\mathbf{x}_{\mathrm{eq}_{3}}^{*}$ and goes to $P_{1}$. In the same way, the blue orbit starting close to $\mathbf{x}_{\mathrm{eq}_{3}}^{*}$ cannot reach $\mathbf{x}_{\mathrm{eq}_{2}}^{*}$ and goes to $P_{4}$. Then there is no heteroclinic orbit from $\mathbf{x}_{\mathrm{eq}_{2}}^{*}$ to $\mathbf{x}_{\mathrm{eq}_{3}}^{*}$, nor vice versa.

The open interval $\Gamma$ is given as $\Gamma=\left(\gamma_{L}, \gamma_{U}\right)$, where

$$
\begin{aligned}
& \gamma_{L}=\alpha\left(1-e^{-a \tau} \cos (b \tau)\right) \approx 1.8826170015164836, \\
& \gamma_{U}=\frac{\alpha\left(e^{-a \tau} \cos (b \tau)-1\right)}{e^{-a \tau} \cos (b \tau)} \approx 2.1329942639693464 .
\end{aligned}
$$

The four cases mentioned generate three types of systems determined by $\gamma$ and $\Gamma$. For instance, for $\gamma=2 \in \Gamma$ and $\alpha=1$ 


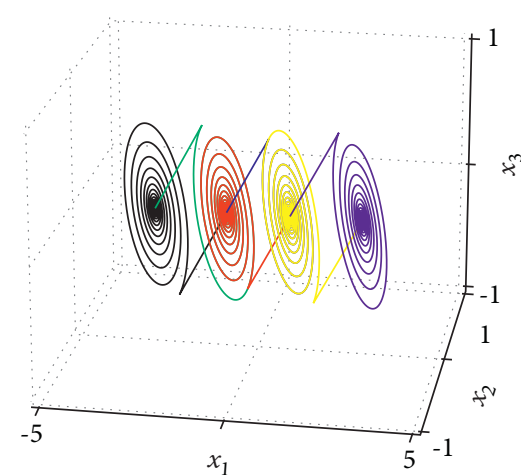

(a)

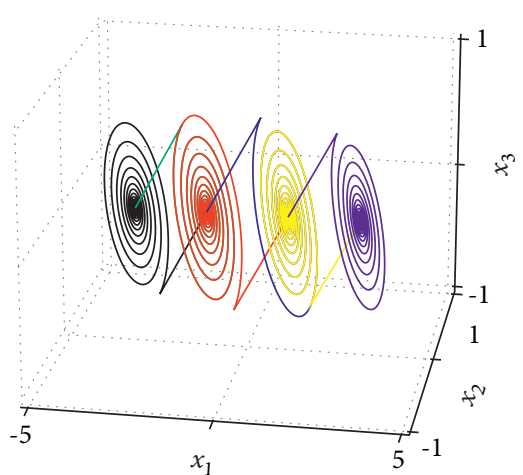

(b)

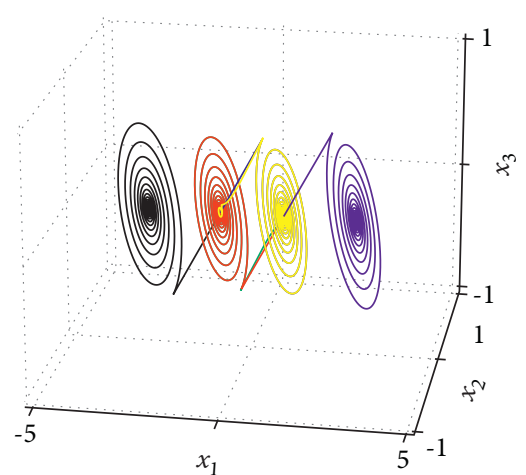

(c)

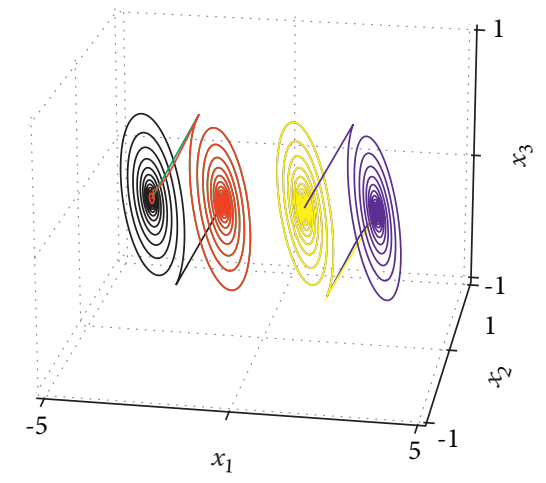

(d)

Figure 3: Heteroclinic orbits of the system given by (1), (3), (5), (13), and (15) for the parameters $a=0.2, b=5, c=-3, \alpha=1$ and different values of $\gamma$. There are six heteroclinic orbits for: (a) $\gamma_{1}=\alpha\left(1-e^{-a \tau} \cos (b \tau)\right)+.00001$, and (b) $\gamma_{2}=\left(\alpha\left(e^{-a \tau} \cos (b \tau)-1\right) / e^{-a \tau} \cos (b \tau)\right)$ -.00001. Four heteroclinic orbits for $(\mathrm{c}) \gamma_{L}=\alpha\left(1-e^{-a \tau} \cos (b \tau)\right)$, and $(\mathrm{d}) \gamma_{U}=\left(\alpha\left(e^{-a \tau} \cos (b \tau)-1\right) / e^{-a \tau} \cos (b \tau)\right)$.

corresponds to the above first and second cases. Then the system presents six heteroclinic orbits which comprise three heteroclinic loops between equilibria: $\mathbf{x}_{\mathrm{eq}_{1}}^{*}$ and $\mathbf{x}_{\mathrm{eq}_{2}}^{*} ; \mathbf{x}_{\mathrm{eq}_{2}}^{*}$ and $\mathbf{x}_{\mathrm{eq}_{3}}^{*} ; \mathbf{x}_{\mathrm{eq}_{3}}^{*}$ and $\mathbf{x}_{\mathrm{eq}_{4}}^{*}$. For $\gamma=1.5<\gamma_{L}$, then $\gamma \notin \Gamma$, and this case corresponds to the above third case. So there are four heteroclinic orbits, and two of them comprise a heteroclinic loop between equilibria $\mathbf{x}_{\mathrm{eq}_{2}}^{*}$ and $\mathbf{x}_{\mathrm{eq}_{3}}^{*}$. For $\gamma_{U}<\gamma=3$, then $\gamma \notin \Gamma$, and this case corresponds to the above fourth case. So there are four heteroclinic orbits that comprise two heteroclinic loops, but now between equilibria: $\mathbf{x}_{\mathrm{eq}_{1}}^{*}$ and $\mathbf{x}_{\mathrm{eq}_{2}}^{*} ; \mathbf{x}_{\mathrm{eq}}^{*}$ and $\mathbf{x}_{\text {eq }}^{*}$. The above three cases generate self-excited attractors as shown below:.

(1) For $\gamma=2 \in \Gamma$, the system presents a self-excited attractor with four scrolls which are shown in Figure 4(a), and its corresponding three heteroclinic loops are shown in Figure 4(d). According to [30], a scroll attractor can be considered a multiscroll attractor when it has at least three scrolls. Thus the attractor shown in Figure 4(a) is a multiscroll attractor. The scrolls are generated around each equilibrium point of the system $\mathbf{x}_{\mathrm{eq}_{i}}^{*}$, with $i=1,2,3,4$.

(2) For $\gamma=3, \gamma>\gamma_{U}$, then $\gamma \notin \Gamma$. The system presents bistability; the two double-scroll self-excited attractors are shown in Figure 4(b). In this case, two heteroclinic orbits are lost, the system exhibits four heteroclinic orbits, i.e., two heteroclinic loops, as shown in Figure 4(e). One double-scroll self-excited attractor oscillates around equilibria $\mathbf{x}_{\mathrm{eq}_{1}}^{*}$ and $\mathbf{x}_{\mathrm{eq}_{2}}^{*}$, while the other self-excited attractor oscillates around equilibria $\mathbf{x}_{\mathrm{eq}_{3}}^{*}$ and $\mathbf{x}_{\mathrm{eq}_{4}}^{*}$. The basin of attraction of each self-excited attractor has surrounded both attractors.

(3) For $\gamma=1.5, \gamma<\gamma_{L}$, then $\gamma \notin \Gamma$. The system presents only one double-scroll self-excited attractor, shown in Figure 4(c). In this case, two heteroclinic orbits are also lost, but only a heteroclinic loop is exhibited. The heteroclinic orbits are shown in Figure 4(f). The double-scroll self-excited attractor oscillates around equilibria $\mathbf{x}_{\mathrm{eq}_{2}}^{*}$ and $\mathbf{x}_{\mathrm{eq}_{3}}^{*}$.

Based on the results reported in [24] about the relation between the location of the symmetric equilibria and the size of the basin of attraction, we could ponder the possible existence of a hidden attractor for the case $\gamma>\gamma_{U}$ because there are oscillations surrounding the two self-excited attractors as a hidden attractor exists. However, the simulations of these systems let us know that hidden attractors are not present. For example, if the $\gamma$ value is increased, then also the distance between the two self-excited attractors increases. This provokes that some initial conditions in the basins of attraction of both attractors generate transitory oscillations resembling a double-scroll attractor. However, 


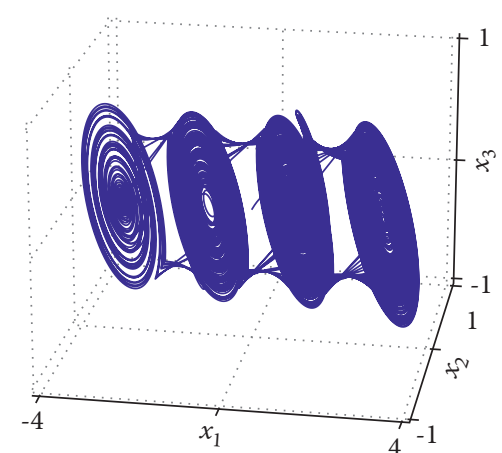

(a)

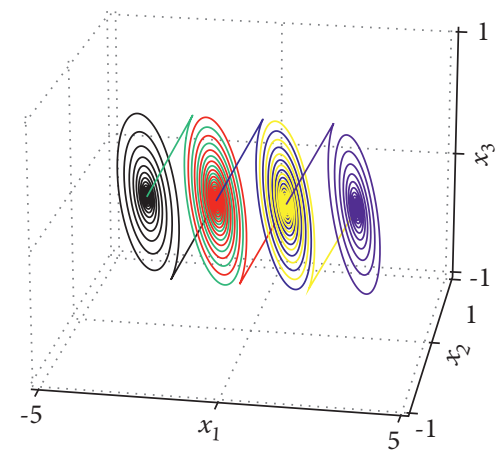

(d)

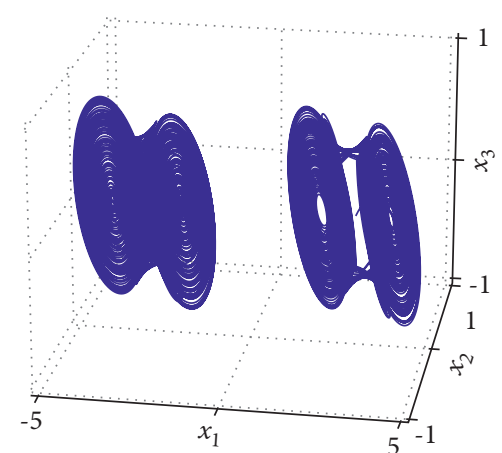

(b)

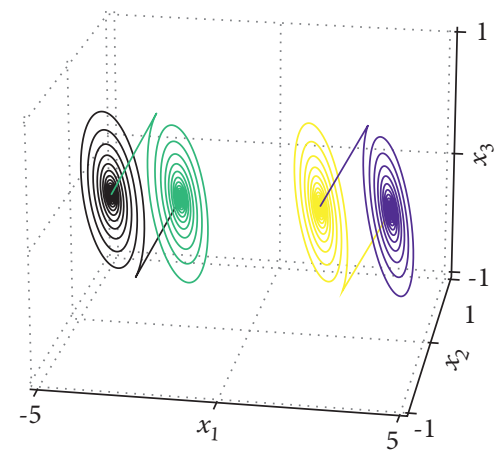

(e)

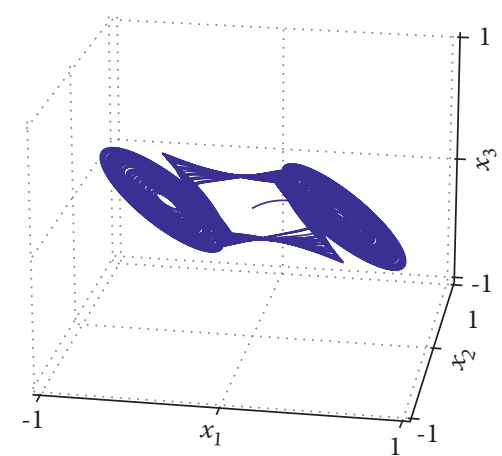

(c)

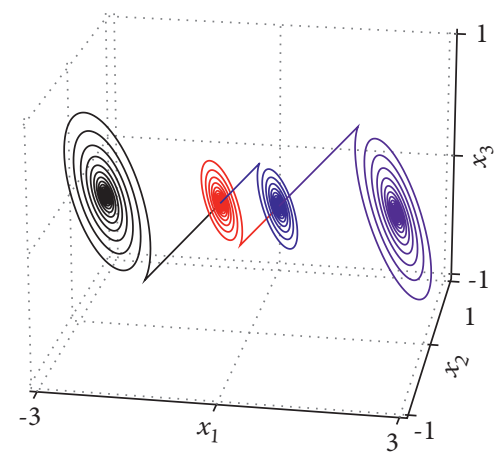

(f)

Figure 4: Attractors and heteroclinic orbits of the system are given by (1), (3), (5), (13), and (15) for $\alpha=1$ and different values of $\gamma$. For $\gamma=2$ the system is exhibited in (a) a quad-scroll attractor, and in (d) six heteroclinic orbits. For $\gamma=3$ the system is exhibited in (b) two doublescroll attractors, and in (e) four heteroclinic orbits. For $\gamma=1.5$ the system is exhibited in (c) a double-scroll attractor and in (f) four heteroclinic orbits.

after some time, these transitory oscillations converge to one of the double-scroll self-excited attractors.

We analyze the trajectory for the initial condition $\mathbf{x}_{0}=$ $(0,0,0)^{T}$ and different values of $\gamma$ fulfilling $\gamma_{U}<\gamma$. The first case is $\gamma=5$ and $t \in[0,40]$ in arbitrary units (a.u). The equilibria are at

$$
\begin{aligned}
& \mathbf{x}_{\mathrm{eq}_{1}}^{*}=\left[\begin{array}{c}
-6 \\
0 \\
0
\end{array}\right], \\
& \mathbf{x}_{\mathrm{eq}_{2}}^{*}=\left[\begin{array}{c}
-4 \\
0 \\
0
\end{array}\right], \\
& \mathbf{x}_{\mathrm{eq}_{3}}^{*}=\left[\begin{array}{l}
4 \\
0 \\
0
\end{array}\right], \\
& \mathbf{x}_{\mathrm{eq}_{4}}^{*}=\left[\begin{array}{l}
6 \\
0 \\
0
\end{array}\right] .
\end{aligned}
$$

Figure 5(a) shows the trajectory which consists of the transitory behavior resembling a double-scroll attractor and after a short time reaches a double-scroll self-excited attractor around equilibria $\mathbf{x}_{\mathrm{eq}_{1}}^{*}$ and $\mathbf{x}_{\mathrm{eq}_{2}}^{*}$.

Increasing the value of $\gamma$ to 15 , the equilibria are located at

$$
\begin{aligned}
& \mathbf{x}_{\mathrm{eq}_{1}}^{*}=\left[\begin{array}{c}
-16 \\
0 \\
0
\end{array}\right], \\
& \mathbf{x}_{\mathrm{eq}_{2}}^{*}=\left[\begin{array}{c}
-14 \\
0 \\
0
\end{array}\right], \\
& \mathbf{x}_{\mathrm{eq}_{3}}^{*}=\left[\begin{array}{c}
14 \\
0 \\
0
\end{array}\right], \\
& \mathbf{x}_{\mathrm{eq}_{4}}^{*}=\left[\begin{array}{c}
16 \\
0 \\
0
\end{array}\right] .
\end{aligned}
$$

And the transitory time to reaches the self-excited attractor is increased. In Figure 5(b), the trajectory is shown for $t \in[0,60]$ a.u. Now, for $\gamma=100$ the equilibria are located at 


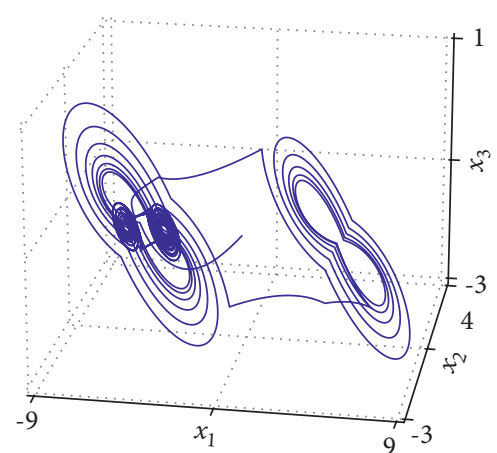

(a)

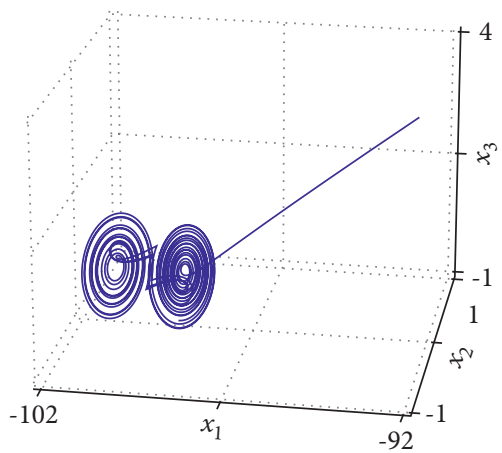

(d)

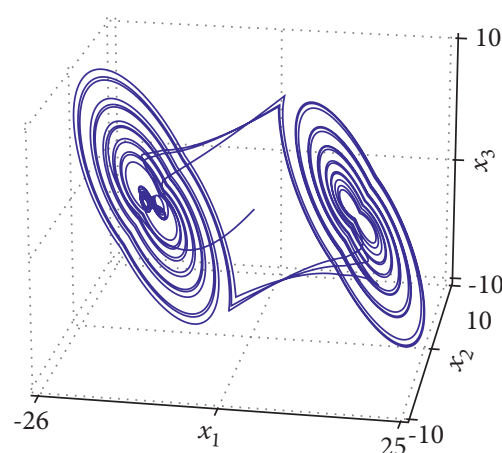

(b)

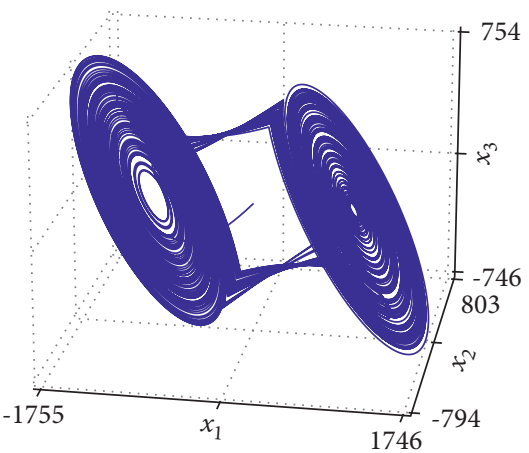

(e)

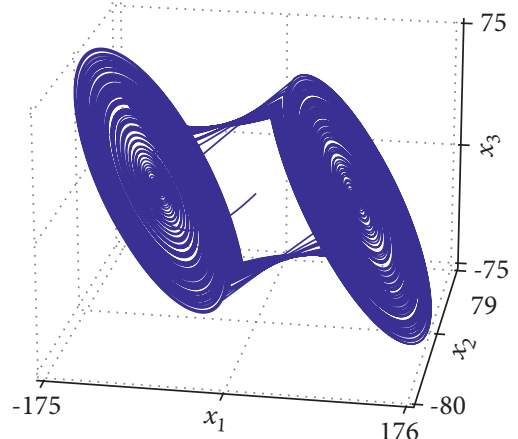

(c)

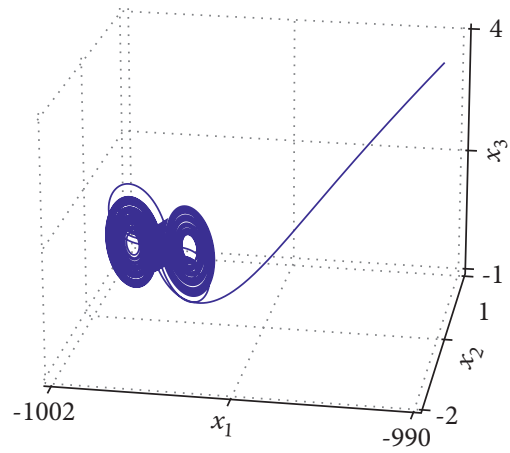

(f)

Figure 5: Trajectory of the system given by (1), (3), (5), (13), and (15) for the initial condition $x=(0,0,0)^{T}, a=0.2, b=5, c=-7, \alpha=1$ and different values of $\gamma$ : (a) $\gamma=5$, the transitory oscillation of double-scroll exhibited and after some time converge to one of the double-scroll self-excited attractors, $t \in[0,40]$ a.u.; (b) $\gamma=15$, the transitory oscillation of double-scroll exhibited and after some time converge to one of the double-scroll self-excited attractors, $t \in[0,60]$ a.u.; (c) $\gamma=100$, the transitory oscillation of double-scroll exhibited for $t \in[0,300]$ a.u.; (d) $\gamma=100$, double-scroll self-excited attractor for $t=[356.6,400]$ a.u.; (e) $\gamma=1000$, the transitory oscillation of double-scroll exhibited for $t \in[0,300]$ a.u.; (f) $\gamma=1000$, the double-scroll self-excited attractor for $t=[3091,3200]$ a.u.

$$
\begin{aligned}
& \mathbf{x}_{\mathrm{eq}_{1}}^{*}=\left[\begin{array}{c}
-101 \\
0 \\
0
\end{array}\right], \\
& \mathbf{x}_{\mathrm{eq}_{2}}^{*}=\left[\begin{array}{c}
-99 \\
0 \\
0
\end{array}\right], \\
& \mathbf{x}_{\mathrm{eq}_{3}}^{*}=\left[\begin{array}{c}
99 \\
0 \\
0
\end{array}\right], \\
& \mathbf{x}_{\mathrm{eq}_{4}}^{*}=\left[\begin{array}{c}
101 \\
0 \\
0
\end{array}\right] .
\end{aligned}
$$

The transitory time lasts longer for the same initial condition. Figure 5(c) shows the transitory oscillations of the trajectory for $t \in[0,300]$ a.u. After a long time, the trajectory reaches a double-scroll self-excited attractor around equilibria $\mathbf{x}_{\mathrm{eq}_{1}}^{*}$ and $\mathbf{x}_{\mathrm{eq}_{2}}^{*}$; see Figure 5(d) for $t \in[356.6,400]$ a.u. Continuing to increase the value to $\gamma=1000$, then this sets the equilibria at

$$
\begin{aligned}
& \mathbf{x}_{\mathrm{eq}_{1}}^{*}=\left[\begin{array}{c}
-1001 \\
0 \\
0
\end{array}\right], \\
& \mathbf{x}_{\mathrm{eq}_{2}}^{*}=\left[\begin{array}{c}
-999 \\
0 \\
0
\end{array}\right], \\
& \mathbf{x}_{\mathrm{eq}_{3}}^{*}=\left[\begin{array}{c}
999 \\
0 \\
0
\end{array}\right], \\
& \mathbf{x}_{\mathrm{eq}_{4}}^{*}=\left[\begin{array}{c}
1001 \\
0 \\
0
\end{array}\right] .
\end{aligned}
$$

Figure 5(e) shows the transitory oscillation of the trajectory when $t \in[0,300]$ a.u., again, transitory time increases, and after this long time, the trajectory again reaches a double-scroll self-excited attractor around equilibria $\mathbf{x}_{\mathrm{eq}_{1}}^{*}$ and $\mathbf{x}_{\mathrm{eq}_{2}}^{*}$, see $5 \mathrm{f}$ for $t \in[3091,3200]$ a.u.

In brief, for $\gamma=5 \mathrm{it}$ took the trajectory around $35 \mathrm{a}$ a.u. To converge to a self-excited attractor, for $\gamma=15$ around 50 a.u. 
To converge, for $\gamma=100$ around 350 a.u. And for $\gamma=1000$ around 3090 a.u. Thus, transitory time seems to increase for some initial conditions when $\gamma$ increases. In all the cases, the trajectories reach a self-excited attractor.

\section{Route to a Self-Excited Attractor}

In this section, the transitory behavior presented in the previous section is studied in order to visualize the route of the transitory double scroll to a self-excited attractor. The idea is to estimate two regions $R_{1}, R_{2} \subset \mathrm{SW}_{23}$, such that any trajectory $\varphi\left(x_{0}\right)$, with $x_{0} \in R_{1} \cup R_{2}$, will eventually go to the self-excited attractor $A_{\text {self } 1}$ or $A_{\text {self } 2}$. These regions are symmetric with respect to the origin and are crossed by the trajectories of the transitory double scroll.

Consider the point $p a$ and its symmetric point $p c=-p a$ :

$$
\begin{gathered}
p a=\left(\begin{array}{c}
-\frac{\gamma-\alpha}{3} \\
0 \\
-\frac{2(\gamma-\alpha)}{3}
\end{array}\right), \\
p c=\left(\begin{array}{c}
\frac{\gamma-\alpha}{3} \\
0 \\
\frac{2(\gamma-\alpha)}{3}
\end{array}\right) .
\end{gathered}
$$

$$
(2,0,-1)\left(\begin{array}{ccc}
\frac{a}{3}+\frac{2 c}{3} & b & \frac{2 c}{3}-\frac{2 a}{3} \\
-\frac{b}{3} & a & \frac{2 b}{3} \\
\frac{c}{3}-\frac{a}{3} & -b & \frac{2 a}{3}+\frac{c}{3}
\end{array}\right)\left(\begin{array}{c}
\frac{x_{3}}{2}-x_{\mathrm{leq}_{i}} \\
x_{2} \\
x_{3}
\end{array}\right)=-(a+c) x_{1 \mathrm{eq}_{i}}+\frac{3(c-a)}{2} x_{3}+3 b x_{2}=0 .
$$

It follows that

$$
x_{2}=\frac{(a+c)}{3 b} x_{\operatorname{leq}_{i}}-\frac{(c-a)}{2 b} x_{3}, \quad \text { with } i=1,2 .
$$

For the vector field of $P_{2}, x_{1 \mathrm{leq}_{2}}=-\gamma+\alpha$, then

$$
x_{2}=\frac{(a+c)}{3 b}(-\gamma+\alpha)-\frac{(c-a)}{2 b} x_{3},
$$

while for the vector field of $P_{3}, x_{\text {leq }_{3}}=\gamma-\alpha$ :
These points are the intersections of the local manifolds in $\mathrm{SW}_{23}$ and are shown in Figure 6.

The trajectories with initial conditions in the points $p a, p c \in \mathrm{SW}_{23}$ converge to equilibria $\mathbf{x}_{\mathrm{eq}_{3}}$ and $\mathbf{x}_{\mathrm{eq}}$, respectively. So, the transient oscillation of the trajectory that resembles a double-scroll attractor interferes when the trajectory reaches neighborhoods $N(p a) \subset \mathrm{SW}_{23}$ and $N(p c) \subset S_{23}$ around $p a$ or $p c$, respectively, because each trajectory with initial condition in $N(p a)$ or $N(p c)$ is led to one of the self-excited attractors $A_{\text {self } 1}$ or $A_{\text {self } 2}$, respectively. So, the aim is to visualize the route to a self-excited attractor when $N(p a) \cap R_{1} \neq \varnothing$ and $N(p c) \cap R_{2} \neq \varnothing$.

Thus, the study in this section has the following structure: First, two regions $R_{1}$ and $R_{2}$ are estimated. The regions are then evaluated numerically to verify their validity. Finally, based on the geometry of the system and the observation of the simulations, conjecture about the necessary conditions for the existence of the hidden attractor.

To simplify the study, some assumptions are made to restrict the systems to a subset of the class.

Assumption 1. The parameter values fulfill the following relations: $(b / a) \geq 25,2 \geq|c / b| \geq(7 / 5)$ and $(\gamma / \alpha) \geq 10$.

To start the analysis, let us find the points in $\mathrm{SW}_{23}$ where the vector fields of $P_{2}$ and $P_{3}$ are tangent to the plane $\mathrm{SW}_{23}$. These points will be called tangent points and can be found from the following equation:

$$
x_{2}=\frac{(a+c)}{3 b}(\gamma-\alpha)-\frac{(c-a)}{2 b} x_{3} \text {. }
$$

According to (16), if $x_{3}>0$ then $\mathrm{SW}_{23}$ belongs to $P_{2}$, and the tangent points to consider in $\mathrm{SW}_{23}$ for $x_{3}>0$ are given by (56). And if $x_{3} \leq 0$, then $\mathrm{SW}_{23}$ belongs to $P_{3}$ and the tangent points are given by (57). An illustration of the tangent points in $\mathrm{SW}_{23}$ is shown in Figure 7, where the points for $P_{2}$ are indicated by a dotted line, while for $P_{3}$ are drawn as a continuous line. 


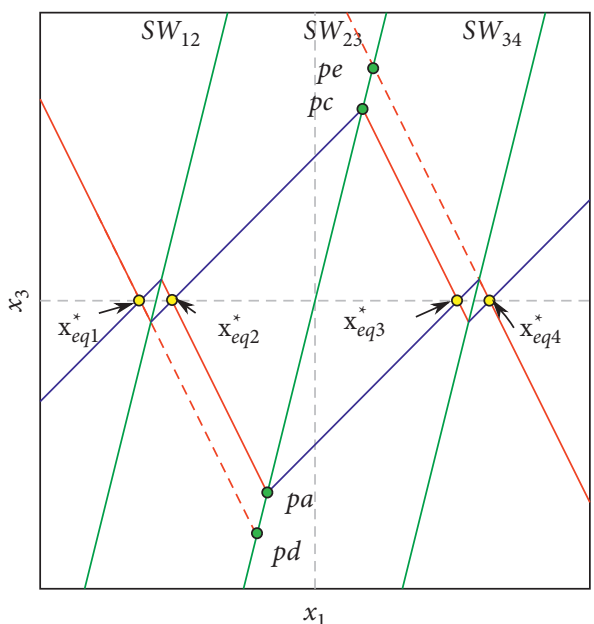

(a)

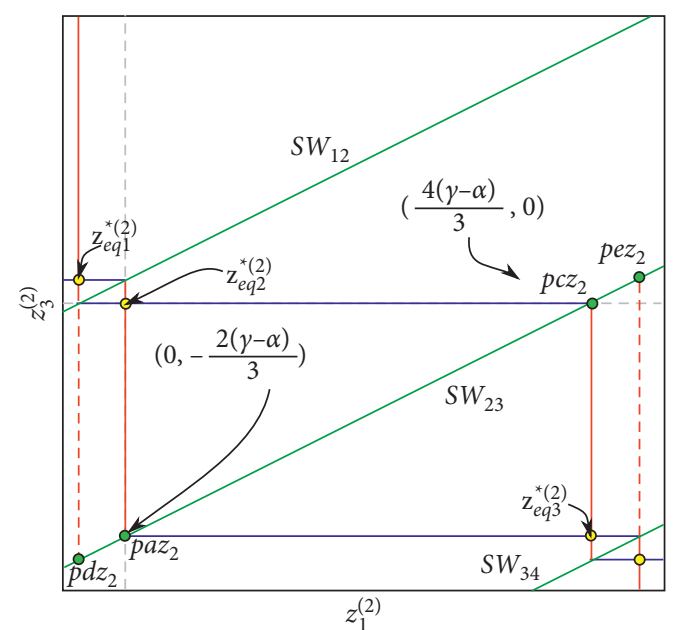

(b)

Figure 6: Projection of the manifolds on (a) $\left(x_{1}-x_{3}\right)$ plane and (b) $\left(z_{1}^{(2)}-z_{3}^{(2)}\right)$ plane. The stable and unstable manifolds are marked with blue and red solid lines, respectively, the switching surfaces with green lines.

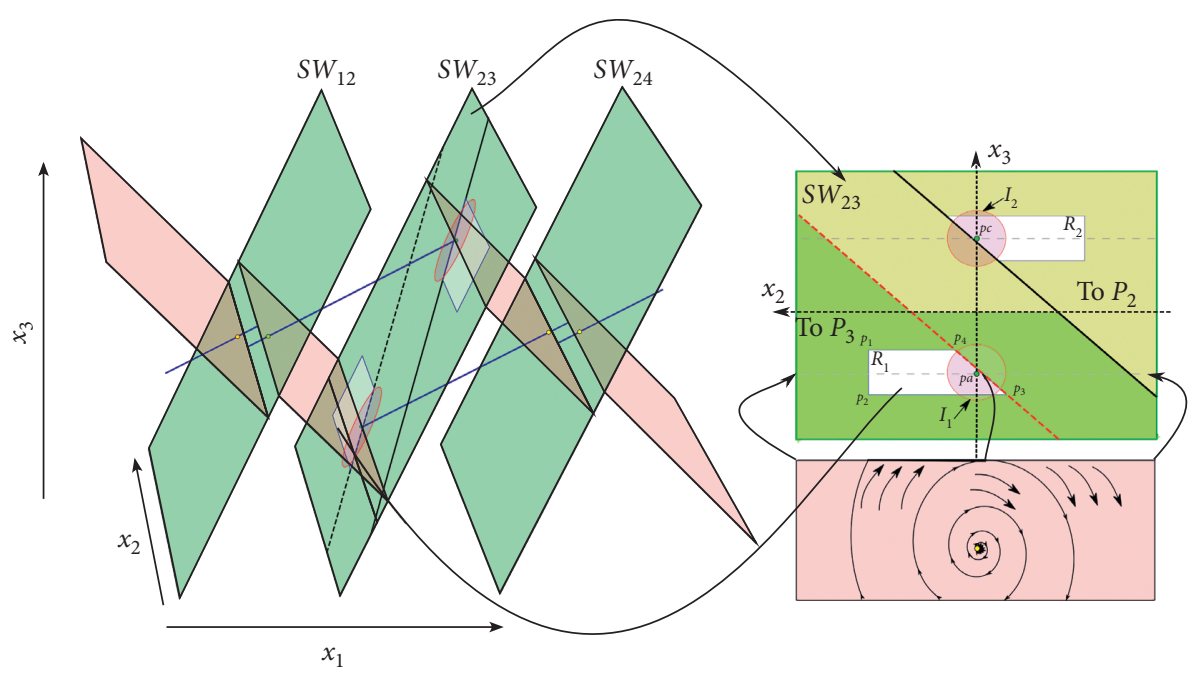

Figure 7: Illustration of the local manifold of the system. Stable manifolds are in blue, unstable manifold in red, and switching surfaces in green. The points where the vector field of $P_{2}$ is tangent to $S W_{23}$ are in a red dotted line, while the points where the vector field of $P_{3}$ are tangent to $S W_{23}$ are in a continuous black line. The symmetric regions $R_{1}$ and $R_{2}$ are in white.

As a starting point to propose the region $R_{1}$ defined by four points $p_{1}, \ldots, p_{4}$, consider the point in $\mathrm{cl}\left(W_{\mathbf{x}_{\mathrm{eq}}}^{u}\right) \cap S W_{23}$ given by (19) that fulfills (56):

$$
p t_{1}=\left(\begin{array}{c}
-\frac{\gamma-\alpha}{3} \\
-\frac{2 a(\gamma-\alpha)}{3 b} \\
-\frac{2(\gamma-\alpha)}{3}
\end{array}\right),
$$

and in $\mathbf{z}^{(2)}$ coordinates

$$
p t_{1} z_{2}=\left(\begin{array}{c}
0 \\
\frac{2 a(\gamma-\alpha)}{3 b} \\
-\frac{2(\gamma-\alpha)}{3}
\end{array}\right) .
$$

The points in $\mathbf{z}^{(2)}$ coordinate system are denoted with the suffix $z_{2}$. If we evaluate the trajectory with an initial condition in $x_{0}=p t_{1} z_{2}$, under the vector field of $P_{2}$ and ignoring the effect of the vector field of $P_{1}$ and $P_{3}$, reaches the point $p t_{2} z_{2} \in \mathrm{SW}_{23}$. The flow $\varphi$ could go from $P_{2}$ to $P_{3}$ through the segment $\overline{p t_{1} z_{2} p t_{2} z_{2}}$. Thus, trajectories with 
initial condition are close to $A_{\text {self } 1}$ but not in the attractor cross $\mathrm{SW}_{23}$ close to the segment $\overline{p t_{1} z_{2} p t_{2} z_{2}}$ then $R_{1}$ should include this segment. However, when the vector field of all atoms is considered, trajectories with initial conditions close to $p t_{1} z_{2}$ could reach $\mathrm{SW}_{23}$ in points whose second component in $z^{(2)}$ coordinates are further from 0 than the second component in $z^{(2)}$ coordinates of $p t_{2} z_{2}$. Thus, let us propose the region $R_{1}$ based on a larger segment $\overline{p a_{1} z_{2} p a_{2} z_{2}}$ such that $\overline{p t_{1} z_{2} p t_{2} z_{2}} \subset \overline{p a_{1} z_{2} p a_{2} z_{2}}$. Consider the initial condition $p a z_{2}$ given in $\mathbf{z}^{(2)}$ coordinates by

$$
p a z_{2}=\left(\begin{array}{c}
0 \\
0 \\
-\frac{2(\gamma-\alpha)}{3}
\end{array}\right),
$$

then, the radius with respect to $\mathbf{z}_{\mathrm{eq}_{2}}^{*}$ would be $(2(\gamma-\alpha) / 3)$. Remember that only the vector field of $P_{2}$ is considered and the trajectory rotates around the axis $z_{1}^{(2)}$. Let us think in an imaginary and impossible case when a trajectory with an initial condition in $p a z_{2}$ rotates around the axis $z_{1}^{(2)}$ and reaches $\mathrm{SW}_{23}$, but instead of the normal increment of radius, let us imagine that the increment in radius corresponds to an evolution time $t=2 \pi / b\left(360^{\circ}\right)$. Thus, the $z_{2}^{(2)}$ component of this point is further from 0 than the $z_{2}^{(2)}$ component of $p t_{2} z_{2}$. Then, we could take $p a_{2} z_{2}=p t_{1} z_{2}$ and find the $z_{2}^{(2)}$ component of $p a_{1} z_{2}$ from

$$
\sqrt{\left(\frac{2(\gamma-\alpha)}{3} e^{a(2 \pi / b)}\right)^{2}-\left(\frac{2(\gamma-\alpha)}{3}\right)^{2}}
$$

By using Assumption 1,

$$
\sqrt{\left(\frac{2(\gamma-\alpha)}{3} e^{a(2 \pi / b)}\right)^{2}-\left(\frac{2(\gamma-\alpha)}{3}\right)^{2}} \leq \gamma \sqrt{\left(\frac{2}{3} e^{a(2 \pi / b)}\right)^{2}-\left(\frac{2}{3}\right)^{2}} \leq 0.5388 \gamma<\frac{3 \gamma}{5}
$$

Remember that $z_{2}^{(2)}=-x_{2}$, then, the points $p a_{1}$ and $p a_{2}$ are given by

$$
\begin{gathered}
p a_{1}=\left(\begin{array}{c}
-\frac{\gamma-\alpha}{3} \\
\frac{3 \gamma}{5} \\
2(\gamma-\alpha) \\
-\frac{\gamma}{3}
\end{array}\right), \\
p a_{2}=\left(\begin{array}{c}
-\frac{\gamma-\alpha}{3} \\
-\frac{2 a(\gamma-\alpha)}{3 b} \\
-\frac{2(\gamma-\alpha)}{3}
\end{array}\right),
\end{gathered}
$$

where $-(2 a(\gamma-\alpha) / 3 b)$ is the tangent coordinate given by (56) for $x_{3}=-(2(\gamma-\alpha) / 3)$.

So, let us propose the region $R_{1}$ delimited by the following four points:

$$
\begin{aligned}
& p_{1}=p a_{1}+\left(\frac{\gamma}{10}, 0, \frac{\gamma}{5}\right)^{T}, \\
& p_{2}=p a_{1}-\left(\frac{\gamma}{10}, 0, \frac{\gamma}{5}\right)^{T}, \\
& p_{3}=p a_{2}+\left(-\frac{\gamma}{10},-\frac{(c-a)(-\gamma / 5)}{2 b},-\frac{\gamma}{5}\right)^{T}, \\
& p_{4}=p a_{2}+\left(\frac{\gamma}{10},-\frac{(c-a)(\gamma / 5)}{2 b}, \frac{\gamma}{5}\right)^{T} .
\end{aligned}
$$

Because $R_{2}$ is symmetric to $R_{1}$ with respect to the origin, then the symmetric region $R_{2}$ is delimited by the points:

$$
\begin{aligned}
& q_{1}=-p_{1}, \\
& q_{2}=-p_{2}, \\
& q_{3}=-p_{3}, \\
& q_{4}=-p_{4} .
\end{aligned}
$$

These regions $R_{1}$ and $R_{2}$ have been proposed taking into consideration that points $p_{d}$ and $p_{e}$ shown in Figure 6(b) are part of the regions. In Figure $8, R_{1}$ and $R_{2}$ are shown in $\mathbf{z}^{(2)}$ coordinates.

Now, let us analyze some scenarios on these regions to see if the proposed regions are good candidates, at least in an 


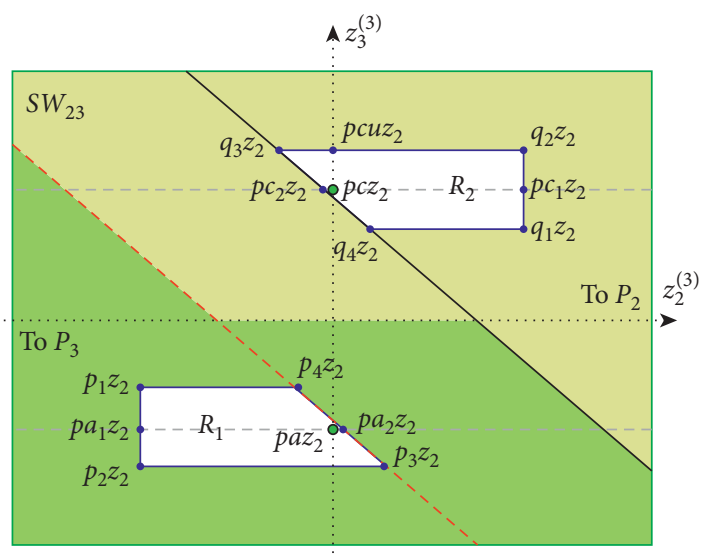

Figure 8: Regions $R_{1}$ and $R_{2}$ on the projection $z_{2}^{(2)}-z_{3}^{(2)}$.

estimated manner. In order to simplify the scenarios, in the following, it is considered that trajectories in $P_{1} \cup P_{2}$ rotate only around the stable manifold of $\mathbf{x}_{\mathrm{eq}_{2}}$, i.e., if only the vector field of $P_{2}$ is considered.

Let us define the set $R_{1} b$ as follows:

$$
R_{1} b=\left\{\mathbf{z}^{(2)} \in \mathbb{R}^{3}: z_{1}^{(2)} \in\left[-\frac{\gamma}{5}, \frac{\gamma}{5}\right]\right\} .
$$

First, let us verify that the points in $R_{2}$ go to $R_{1} b$.

The evaluation of the vector field in $p c_{1} z_{2}$ tells us that the spin is counterclockwise in $\mathbf{z}^{(2)}$ coordinates. From Figures 8 and $6(\mathrm{~b})$, it is not hard to see that the points below the segment $\overline{p c z_{2} p c_{1} z_{2}}$ produce trajectories that can perform a turn of $\pi$ around the $z_{1}^{(2)}$ axis without reaching SW $\mathrm{SW}_{23}$ again. The time that corresponds to a turn of $2 \pi$ is $T=2 \pi / b$. The point $p c_{1} z_{2}$ is given by

$$
p c_{1} z_{2}=\left(\begin{array}{c}
\frac{4(\gamma-\alpha)}{3} \\
\frac{3 \gamma}{5} \\
0
\end{array}\right) .
$$

Consider the trajectory with an initial condition in $p c_{1} z_{2}$, and an evolution time that corresponds to a turn of $\pi$ around the $z_{1}^{(2)}$ axis. After this time, the first component of the state vector can be found from

$$
z_{1}^{(2)}=\left(\frac{4(\gamma-\alpha)}{3}\right) e^{c(\pi / b)} .
$$

If $z_{1}^{(2)} \leq \gamma / 5$ means that the trajectory with an initial condition in $p c_{1} z_{2}$ reaches $R_{1} b$. Consider Assumption 1 for a big value of $z_{1}^{(2)}$ (when $\gamma$ is too big):

$$
\left(\frac{4(\gamma-\alpha)}{3}\right) e^{c(\pi / b)} \leq \frac{4 \gamma}{3} e^{c(\pi / b)} \leq 0.0164 \gamma<\frac{\gamma}{5}
$$
$R_{1} b$.

Thus the set $\left\{\mathbf{z}^{(2)} \in R_{2}: z_{2}^{(2)} \geq 0, z_{3}^{(2)} \leq 0\right\}$ reaches the set

Now consider the point $q_{2} z_{2}$ given by

$$
q_{2} z_{2}=\left(\begin{array}{c}
\frac{23 \gamma}{15}-\frac{4 \alpha}{3} \\
\frac{3 \gamma}{5} \\
\frac{\gamma}{10}
\end{array}\right)
$$

The angle produced by the radius from the point $q_{2} z_{2}$ to the $z_{1}^{(2)}$ axis and the plane $z_{1}^{(2)}-z_{2}^{(2)}$ is given by

$$
\arctan \left(\frac{\gamma / 10}{3 \gamma / 5}\right)=\arctan \left(\frac{1}{6}\right)=0.1654
$$

Let us consider that the trajectory with the initial condition in $q_{2} z_{2}$ evolves for a duration time that corresponds to $\pi-2(0.1654)=2.8113$. The first component of the state vector after this duration is given by

$$
z_{1}^{(2)}=\left(\frac{23 \gamma}{15}-\frac{4 \alpha}{3}\right) e^{c(2.8113 / b)}
$$

As before, if $z_{1}^{(2)} \leq \gamma / 5$ means that the trajectory with an initial condition in $q_{2} z_{2}$ reaches $R_{1} b$. Consider again Assumption 1 for a big value of $z_{1}^{(2)}$ :

$$
\left(\frac{23 \gamma}{15}-\frac{4 \alpha}{3}\right) e^{c(2.8113 / b)} \leq\left(\frac{23 \gamma}{15}\right) e^{c(2.8113 / b)} \leq 0.0299 \gamma<\frac{\gamma}{5}
$$

Thus, $q_{2} z_{2}$ reaches the region $R_{1} b$. Moreover, since the points in the segment $\overline{q_{2} z_{2} p c_{1} z_{2}}$ produce a radius whose angle with the plane $z_{1}^{(2)}-z_{2}^{(2)}$ is between 0 and 2.8113, the trajectories starting in this segment also reach the set $R_{1} b$.

Now consider the point $p c u z_{2}$ given by

$$
\operatorname{pcuz}_{2}=\left(\begin{array}{c}
\frac{23 \gamma}{15}-\frac{4 \alpha}{3} \\
0 \\
\frac{\gamma}{10}
\end{array}\right) .
$$

The trajectories with the initial condition in the segment $\overline{p c z_{2} p c u z_{2}}$ can turn $\pi / 2$ without reaching $\mathrm{SW}_{23}$. Thus, let us consider that the trajectory with the initial condition in pcuz $_{2}$ evolves for a duration time that corresponds to $\pi / 2$. The first component of the state vector after this duration is given by

$$
z_{1}^{(2)}=\left(\frac{23 \gamma}{15}-\frac{4 \alpha}{3}\right) e^{c(\pi / 2 b)}
$$

If $z_{1}^{(2)} \leq \gamma / 5$ means that the trajectory with an initial condition in $p c u z_{2}$ reaches $R_{1} b$, consider Assumption 1 for a big value of $z_{1}^{(2)}$ :

$$
\left(\frac{23 \gamma}{15}-\frac{4 \alpha}{3}\right) e^{c(\pi / 2 b)} \leq\left(\frac{23 \gamma}{15}\right) e^{c(\pi / 2 b)} \leq 0.17 \gamma<\frac{\gamma}{5}
$$


Thus the trajectories with the initial condition in the set $\left\{\mathbf{z}^{(2)} \in R_{2}: z_{2}^{(2)} \geq 0, z_{3}^{(2)} \geq 0\right\}$ converge to the set $R_{1} b$.

Now consider the point $q_{3} z_{2}$

$$
q_{3} z_{2}=\left(\begin{array}{c}
\frac{23 \gamma}{15}-\frac{4 \alpha}{3} \\
\frac{3 c \gamma-23 a \gamma}{30 b}+\frac{2 a \alpha}{3 b} \\
\frac{\gamma}{10}
\end{array}\right) .
$$

To estimate if that the trajectory starting in $q_{3} z_{2}$ is not going to reach $\mathrm{SW}_{23}$ when the radius form an angle of $3 \pi / 2$ with the plane $z_{1}^{(2)}-z_{2}^{(2)}$, let us consider the following exaggerated scenario: The radius size corresponds to a duration equivalent to $3 \pi / 2$, but the $z_{1}^{(2)}$ component corresponds to a duration equivalent to $\pi / 2$ of oscillation, i.e., when the radius form an angle of $3 \pi / 2$ with the plane $z_{1}^{(2)}-z_{2}^{(2)}$, a smaller radius than the real one is considered; also, a larger value of $z_{1}^{(2)}$ than the real value is considered.

Then to obtain the radius:

$$
r=e^{a(3 \pi / 2 b)} \sqrt{\left(\frac{3 c \gamma-23 a \gamma}{30 b}+\frac{2 a \alpha}{3 b}\right)^{2}+\left(\frac{\gamma}{10}\right)^{2}} .
$$

Under Assumption 1 for the largest radius:

$$
\begin{gathered}
e^{a(3 \pi / 2 b)} \sqrt{\left(\frac{3 c \gamma-23 a \gamma}{30 b}+\frac{2 a \alpha}{3 b}\right)^{2}+\left(\frac{\gamma}{10}\right)^{2}} \leq e^{a(3 \pi / 2 b)} \sqrt{\left(\frac{3 c \gamma-23 a \gamma}{30 b}\right)^{2}+\left(\frac{\gamma}{10}\right)^{2}}, \\
e^{a(3 \pi / 2 b) \sqrt{\left(\frac{3 c \gamma}{30 b}-\frac{23 a \gamma}{30 b}\right)^{2}+\left(\frac{\gamma}{10}\right)^{2}}} \leq e^{a(3 \pi / 2 b)} \sqrt{\left(-\frac{1 \gamma}{5}-\frac{23 \gamma}{750}\right)^{2}+\left(\frac{\gamma}{10}\right)^{2}} \\
e^{a(3 \pi / 2 b)} \sqrt{\left(-\frac{1 \gamma}{5}-\frac{23 \gamma}{750}\right)^{2}+\left(\frac{\gamma}{10}\right)^{2}}=\gamma e^{a(3 \pi / 2 b)} \sqrt{\left(-\frac{173}{750}\right)^{2}+\left(\frac{1}{10}\right)^{2}}, \\
\gamma e^{a(3 \pi / 2 b)} \sqrt{\left(-\frac{173}{750}\right)^{2}+\left(\frac{1}{10}\right)^{2}} \leq 0.3036 \gamma .
\end{gathered}
$$

The value of $z_{1}^{(2)}$ after a duration equivalent to $90^{\circ}$ is given by

$$
z_{1}^{(2)}=\left(\frac{23 \gamma}{15}-\frac{4 \alpha}{3}\right) e^{c(\pi / 2 b)}
$$

Under Assumption 1 for the biggest $z_{1}^{(2)}$

$$
\begin{aligned}
\left(\frac{23 \gamma}{15}-\frac{4 \alpha}{3}\right) e^{c(\pi / 2 b)} & \leq\left(\frac{23 \gamma}{15}\right) e^{c(\pi / 2 b)}, \\
\left(\frac{23 \gamma}{15}\right) e^{c(\pi / 2 b)} & \leq 0.17 \gamma<\frac{\gamma}{5} .
\end{aligned}
$$

In $\mathbf{z}^{(2)}$ coordinates, a specific value of $z_{1}^{(2)} \mathrm{SW}_{23}$ fulfills $z_{3}^{(2)}=-(2(\gamma-\alpha) / 3)+\left(z_{1}^{(2)} / 2\right)$. At this angle of $3 \pi / 2$ the radius is $r=-z_{3}^{(2)}$. Then, if the values found for this scenario fulfill the following inequality, it can be concluded that the trajectory with an initial condition in $q_{3} z_{2}$ does not reach $\mathrm{SW}_{23}$ after a duration that corresponds to an oscillation of $\pi / 2$ :

$$
-0.3036 \gamma>-\frac{2(\gamma-\alpha)}{3}+0.085 \gamma
$$

under Assumption 1 for the worst case

$$
-0.3036 \gamma>-\frac{18 \gamma}{30}+0.085 \gamma=-0.515 \gamma
$$

Then, the trajectory remains for the duration that corresponds to $\pi / 2$. Also, the trajectory does not reach $\mathrm{SW}_{23}$ when the radius is at an angle of $3 \pi / 2$ with the plane $z_{1}^{(2)}-$ $z_{2}^{(2)}$ even when the radius growth is exaggerated. It can be concluded that the trajectory with the initial condition in $q_{3} z_{2}$ could reach $\mathrm{SW}_{23}$ until the second time it approaches $\mathrm{SW}_{23}$ and reaches $R_{1} b$.

Since $q_{3} z_{2}$ is the point in the set $\left\{\mathbf{z}^{(2)} \in R_{2}: z_{2}^{(2)} \leq 0\right\}$ that produces the largest radius of that set, the trajectories with the initial condition in this set also reach $R_{1} b$. Then the trajectories starting at $R_{2}$ reach $R_{1} b$.

Now to estimate if the trajectories that start in $R_{2}$ reach $R_{1} \subset R_{1} b$ is enough to verify the trajectories starting in the segment $\overline{q_{1} z_{2} q_{2} z_{2}}$ since these produce the largest radius in $R_{1} b$.

Consider the points $q_{1} z_{2}$ and $q_{2} z_{2}$ 


$$
\begin{aligned}
& q_{1} z_{2}=\left(\begin{array}{c}
\frac{17 \gamma}{15}-\frac{4 \alpha}{3} \\
\frac{3 \gamma}{5} \\
-\frac{\gamma}{10}
\end{array}\right), \\
& q_{2} z_{2}=\left(\begin{array}{c}
\frac{23 \gamma}{15}-\frac{4 \alpha}{3} \\
\frac{3 \gamma}{5} \\
\frac{\gamma}{10}
\end{array}\right),
\end{aligned}
$$

both points produce the same radius with a different angle. However, more oscillation time before reaching $\mathrm{SW}_{23}$ is expected from $q_{1} z_{2}$. Thus, consider the trajectory with the initial condition in $q_{1} z_{2}$ and the evolution time that corresponds to $3 \pi / 2+0.1651=4.8775$, which is an exaggerated angle since $\mathrm{SW}_{23}$ is reached before that.

$$
r=e^{a(4.8775 / b)} \sqrt{\left(\frac{3 \gamma}{5}\right)^{2}+\left(\frac{\gamma}{10}\right)^{2}}=\gamma e^{a(4.8775 / b)} \sqrt{\frac{37}{100}} .
$$

Under Assumption 1,

$$
\gamma \sqrt{\frac{37}{100}} e^{a(4.8775 / b)} \leq 0.7393 \gamma
$$

Consider the points $p_{1} z_{2}, p a_{1} z_{2}$ and $p_{2} z_{2}$

$$
\begin{aligned}
& p_{1} z_{2}=\left(\begin{array}{c}
\frac{\gamma}{5} \\
-\frac{3 \gamma}{5} \\
\frac{2 \alpha}{3}-\frac{17 \gamma}{30}
\end{array}\right), \\
& p a_{1} z_{2}=\left(\begin{array}{c}
0 \\
3 \gamma \\
-\frac{3 \gamma}{5} \\
-\frac{2(\gamma-\alpha)}{3}
\end{array}\right), \\
& p_{2} z_{2}=\left(\begin{array}{c}
-\frac{\gamma}{5} \\
-\frac{3 \gamma}{5} \\
\frac{2 \alpha}{3}-\frac{23 \gamma}{30}
\end{array}\right) .
\end{aligned}
$$

The minimum radius in the segment $\overline{p a_{1} z_{2}, p a_{2} z_{2}}$ is as follows:

$$
r=\sqrt{\left(\frac{3 \gamma}{5}\right)^{2}+\left(\frac{2 \alpha}{3}-\frac{17 \gamma}{30}\right)^{2}}
$$

Under Assumption 1, the radius from the segment $\overline{p a_{1} z_{2}, p a_{2} z_{2}}$ is as follows:

$$
\begin{gathered}
\sqrt{\left(\frac{3 \gamma}{5}\right)^{2}+\left(\frac{2 \alpha}{3}-\frac{17 \gamma}{30}\right)^{2}} \geq \sqrt{\left(\frac{3 \gamma}{5}\right)^{2}+\left(-\frac{\gamma}{2}\right)^{2}} \\
\gamma \sqrt{\left(\frac{3}{5}\right)^{2}+\left(-\frac{1}{2}\right)^{2}}=0.781 \gamma .
\end{gathered}
$$

Since $0.7393 \gamma<0.781 \gamma$ even when the increment of radius was exaggerated, it is expected that the trajectories with an initial condition in $R_{2}$ reach $R_{1}$ or a self-excited attractor. In the same way, the trajectories with an initial condition in $R_{1}$ reach $R_{2}$ or go to a self-excited attractor.

To verify the region for the parameters $a=0.2, b=5$, $c=-7$ and $\alpha=1$, seven trajectories have been simulated and are shown in Figure 9(a) for $\gamma=10$ and $\gamma=100$ in Figure 9(b).

Now consider two sets of initial conditions in $\mathrm{SW}_{23}, I_{1}$ and $I_{2}$, such that subsets $N(p a)$ and $N(p c)$ of these sets produce trajectories that end in one of the self-excited attractors. These sets are drawn by circles in Figure 7.

It is easy to see that if $\gamma$ increases, then the regions $R_{1}$ and $R_{2}$ grow, but the subsets of initial conditions in $I_{1}$ and $I_{2}$ that reach a self-excited attractor without reaching $S_{23}$ again are reduced.

Let us look at the system in $\mathbf{z}^{(2)}$ coordinates, as $\gamma$ grows, $\mathrm{SW}_{23}$ and $p c z_{2}$ are further from the $\mathbf{z}_{\mathrm{eq} 2}^{*}$ and then it takes more time for the trajectories close to $p c z_{2}$ to travel along the $z_{1}^{(2)}$ direction to get close to $\mathbf{z}_{\mathrm{eq} 2}^{*}$; however, the expansion along $z_{2}^{(2)}$ and $z_{3}^{(2)}$ remains the same; then the subsets of initial conditions that reach the self-excited attractors without reaching $\mathrm{SW}_{23}$ again shrink in $I_{1}$ and $I_{2}$ but never disappear. As $p c$ and $p a$ belong to $R_{1}$ and $R_{2}$, respectively, then there will always be an intersection of these regions $R_{1}$ and $R_{2}$ with the subsets of initial conditions that reaches the self-excited attractors in the regions $I_{1}$ and $I_{2}$.

This explains why as $\gamma$ is increased, it is easy to find initial conditions such that the transitory lasts long. Then we come to the conjecture that a necessary condition for the existence of a hidden attractor is that the intersection of regions $R_{1}$ and $R_{2}$ with those sets given by $I_{1}$ and $I_{2}$ must be empty, i.e., $N(p a) \cap R_{1}=\varnothing$ and $N(p c) \cap R_{2}=\varnothing$.

\section{Emergence of Hidden Attractors}

In this section, a modification is made to the previous class of systems to meet the conjecture requirement. A way to produce $N(p a) \cap R_{1}=\varnothing$ and $N(p c) \cap R_{2}=\varnothing$ and allow the existence of a hidden attractor is by modifying the commutation surface $S W_{23}$ between the two self-excited attractors. Consider the following switching planes: 


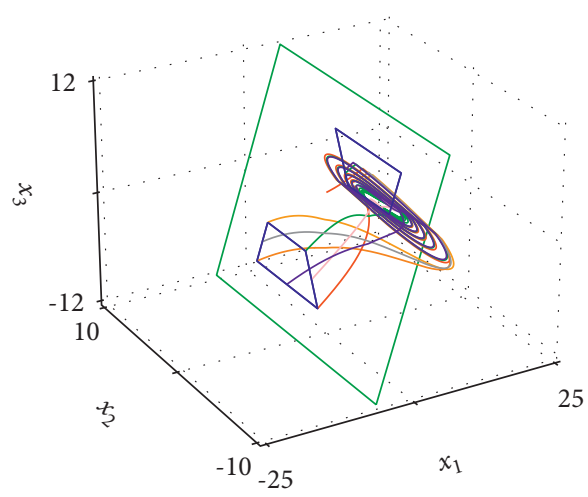

(a)

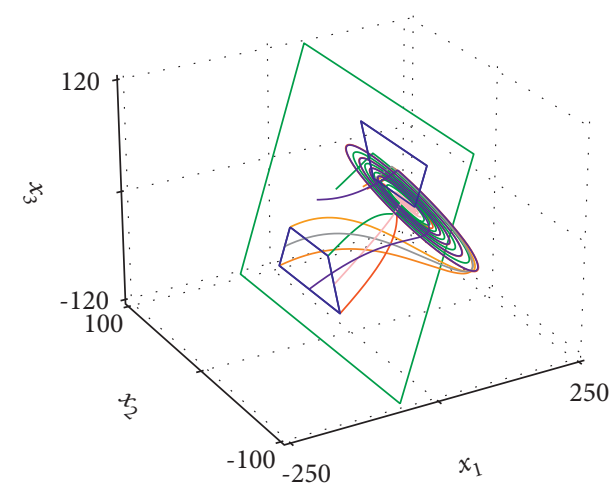

(b)

FIgURE 9: Seven trajectories of the system given by (1), (3), (5), (13), and (15) starting in $R_{1}$ with $a=0.2, b=5, c=-7, \alpha=1$ and different values of $\gamma$ : (a) $\gamma=10$ and (b) $\gamma=100$.

$$
\begin{aligned}
& \mathrm{SW}_{12}=\operatorname{cl}\left(P_{1}\right) \cup \operatorname{cl}\left(P_{2}\right)=\left\{\mathbf{x} \in \mathbb{R}^{3}: 2 x_{1}-x_{3}=-2 \gamma, x_{1}<0\right\}, \\
& \mathrm{SW}_{23}=\operatorname{cl}\left(P_{2}\right) \cup \operatorname{cl}\left(P_{3}\right)=\left\{\mathbf{x} \in \mathbb{R}^{3}: x_{1}=0\right\}, \\
& \mathrm{SW}_{34}=\operatorname{cl}\left(P_{3}\right) \cup \operatorname{cl}\left(P_{4}\right)=\left\{\mathbf{x} \in \mathbb{R}^{3}: 2 x_{1}-x_{3}=2 \gamma, x_{1}>0\right\} .
\end{aligned}
$$

Note that the switching surface $\mathrm{SW}_{23}$ has a new location while the switching surfaces $\mathrm{SW}_{12}$ and $\mathrm{SW}_{34}$ keep their original locations. This new arrangement keeps the existence of the two heteroclinic loops and thus the two self-excited attractors. The new projections of the system in $\mathbf{x}$ and $\mathbf{z}^{(2)}$ coordinates are shown in Figure 10.

To study the emergence of a hidden attractor, the same procedure is followed as in the previous section.

Let us find the points in $\mathrm{SW}_{23}$ where the vector fields of $P_{2}$ and $P_{3}$ are tangent to the plane $\mathrm{SW}_{23}$. These points can be found from the following equation:

$$
(1,0,0)\left(\begin{array}{ccc}
\frac{a}{3}+\frac{2 c}{3} & b & \frac{2 c}{3}-\frac{2 a}{3} \\
-\frac{b}{3} & a & \frac{2 b}{3} \\
\frac{c}{3}-\frac{a}{3} & -b & \frac{2 a}{3}+\frac{c}{3}
\end{array}\right)\left(\begin{array}{c}
-x_{\mathrm{eq}_{i}} \\
x_{2} \\
x_{3}
\end{array}\right)=-x_{\mathrm{eq}_{i}} \frac{(a+2 c)}{3}+b x_{2}+\frac{2 c-2 a}{3} x_{3}=0
$$

$$
x_{2}=x_{\mathrm{eq}_{i}} \frac{(a+2 c)}{3 b}-\frac{2 c-2 a}{3 b} x_{3}, \quad \text { with } i=1,2 .
$$

Then for the vector field of $P_{2}$ we have the following expression:

$$
x_{2}=\frac{-(\gamma-\alpha)(a+2 c)}{3 b}-\frac{2 c-2 a}{3 b} x_{3} .
$$

For the vector field of $P_{3}$ the expression is as follows:

$$
x_{2}=\frac{(\gamma-\alpha)(a+2 c)}{3 b}-\frac{2 c-2 a}{3 b} x_{3} \text {. }
$$

Consider the point in $\mathrm{cl}\left(W_{\mathbf{x}_{\mathrm{eq}_{2}}}^{u} \cap \mathrm{SW}_{23}\right)$ that fulfills (92): and in $\mathbf{z}^{(2)}$ coordinates

$$
p t_{1}=\left(\begin{array}{c}
0 \\
-\frac{a(\gamma-\alpha)}{b} \\
-(\gamma-\alpha)
\end{array}\right)
$$

$$
p t_{1} z_{2}=\left(\begin{array}{c}
0 \\
\frac{a(\gamma-\alpha)}{b} \\
-(\gamma-\alpha)
\end{array}\right) .
$$




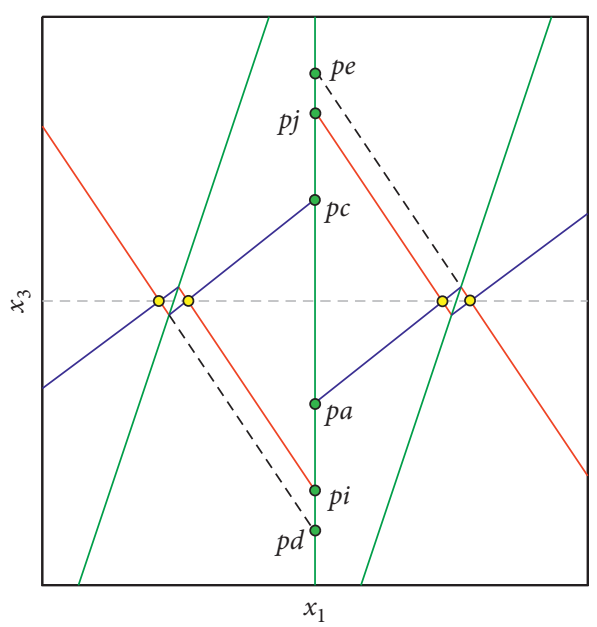

(a)

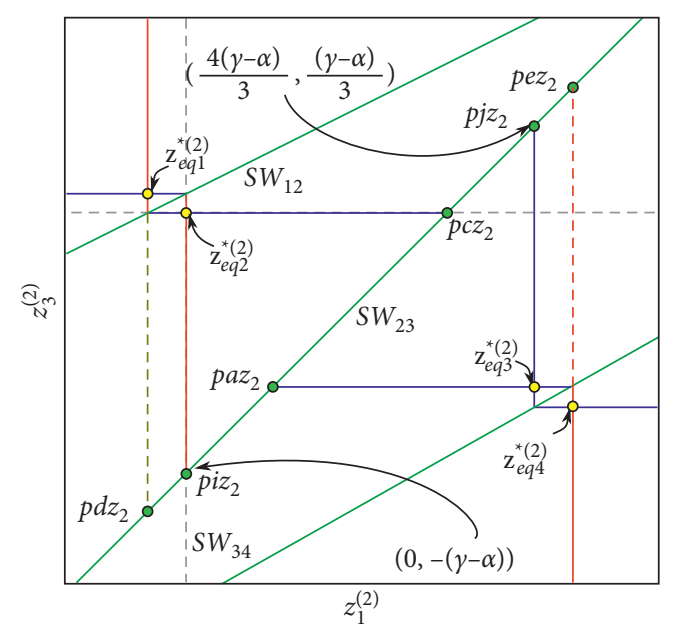

(b)

FIGURe 10: Projection of the manifolds on (a) $x_{1}-x_{3}$ and (b) $z_{1}^{(2)}-z_{3}^{(2)}$. The stable and unstable manifolds are marked with blue and red solid lines, respectively, the switching surfaces with green lines.

If we evaluate the trajectory with initial condition in $x_{0}=p t_{1} z_{2}$, under the vector field of $P_{2}$ ignoring the effect of the vector field of $P_{1}$ and $P_{3}$, reaches the point $p t_{2} z_{2} \in \mathrm{SW}_{23}$. The flow $\varphi$ could go from $P_{2}$ to $P_{3}$ through the segment $\overline{p t_{1} z_{2} p t_{2} z_{2}}$. Thus, trajectories with initial condition close to $A_{\text {self } 1}$ but not in the attractor cross $\mathrm{SW}_{23}$ close to the segment $p t_{1} z_{2} p t_{2} z_{2}$, then, $R_{1}$ should include this segment. However, when the vector field of all atoms is considered, trajectories with initial conditions close to $p t_{1} z_{2}$ could reach $\mathrm{SW}_{23}$ in points whose second component in $z^{(2)}$ coordinates is further from 0 than the second component in $z^{(2)}$ coordinates of $p t_{2} z_{2}$. This allows us to propose the region $R_{1}$ based on a larger segment $\overline{p i_{1} z_{2} p i_{2} z_{2}}$ such that $\overline{p t_{1} z_{2} p t_{2} z_{2}} \subset \overline{p i_{1} z_{2} p i_{2} z_{2}}$. Consider the initial condition piz $_{2}$ given in $\mathbf{z}^{(2)}$ coordinates by

$$
p i z_{2}=\left(\begin{array}{c}
0 \\
0 \\
-(\gamma-\alpha)
\end{array}\right),
$$

then, the radius with respect to $\mathbf{z}_{\mathrm{eq}_{2}}^{*}$ would be $(\gamma-\alpha)$. Remember that only the vector field of $P_{2}$ is considered and the trajectory rotates around the axis $z_{1}^{(2)}$. Let us think in an imaginary and impossible case when a trajectory with an initial condition in $p i z_{2}$ rotates around the axis $z_{1}^{(2)}$ and reaches $S W_{23}$, but instead of the normal increment of radius, let us imagine that the increment in radius corresponds to an evolution time $t=2 \pi / b\left(360^{\circ}\right)$. Thus, the $z_{2}^{(2)}$ component of this point is further from 0 than the $z_{2}^{(2)}$ component of $p t_{2} z_{2}$. Then, we could take $p i_{2} z_{2}=p t_{1} z_{2}$ and find the $z_{2}^{(2)}$ component of $p a_{1} z_{2}$ from

$$
\sqrt{\left(e^{a(2 \pi / b)}(\gamma-\alpha)\right)^{2}-(\gamma-\alpha)^{2}} .
$$

Consider Assumption 1, then,

$$
\sqrt{\left(e^{a(2 \pi / b)}(\gamma-\alpha)\right)^{2}-(\gamma-\alpha)^{2}} \leq \gamma \sqrt{\left(e^{a(2 \pi / b)}\right)^{2}-1} \leq 0.80815 \gamma \approx \frac{4 \gamma}{5} .
$$

Remember that $z_{2}^{(2)}=-x_{2}$, then, the points $p i_{1}$ and $p i_{2}$ are given by

$$
\begin{aligned}
& p i_{1}=\left(\begin{array}{c}
0 \\
4 \gamma \\
5 \\
-(\gamma-\alpha)
\end{array}\right), \\
& p i_{2}=\left(\begin{array}{c}
0 \\
-\frac{a(\gamma-\alpha)}{b} \\
-(\gamma-\alpha)
\end{array}\right),
\end{aligned}
$$

where $-(a(\gamma-\alpha) / b)$ is the tangent coordinate given by (92) for $x_{3}=-(\gamma-\alpha)$.

Let us propose a region $R_{1}$ delimited by the following four points:

$$
\begin{aligned}
& p_{1}=p i_{1}+\left(0,0, \frac{\gamma}{5}\right)^{T}, \\
& p_{2}=p i_{1}-\left(0,0, \frac{\gamma}{5}\right)^{T}, \\
& p_{3}=p i_{2}+\left(0,-\left(\frac{2 c-2 a}{3 b}\right)\left(-\frac{\gamma}{5}\right),-\frac{\gamma}{5}\right)^{T}, \\
& p_{4}=p i_{2}+\left(0,-\left(\frac{2 c-2 a}{3 b}\right)\left(\frac{\gamma}{5}\right), \frac{\gamma}{5}\right)^{T} .
\end{aligned}
$$

Then, the symmetric region $R_{2}$ is delimited by the points: 


$$
\begin{aligned}
& q_{1}=-p_{1}, \\
& q_{2}=-p_{2}, \\
& q_{3}=-p_{3}, \\
& q_{4}=-p_{4} .
\end{aligned}
$$

The regions $R_{1}$ and $R_{2}$ have been proposed taking into consideration that $p d$ and $p e$ are part of the regions and $p a$ and $p c$ are not. In Figure $11, R_{1}$ and $R_{2}$ are shown in $\mathbf{z}^{(2)}$ coordinates.

Now, let us analyze some scenarios on these regions to see if the proposed regions are good candidates, at least in an estimated manner. In order to simplify the scenarios, in the following, it is considered that trajectories in $P_{1} \cup P_{2}$ rotate only around the stable manifold of $\mathbf{x}_{\mathrm{eq}_{2}}$, i.e., if only the vector field of $P_{2}$ is considered. Since $p_{1} z_{2}-p i$ and $p i z_{2}-p_{2} z_{2}$ are given by

$$
\begin{gathered}
p_{1} z_{2}-p i=\left(\begin{array}{c}
\frac{2 \gamma}{15} \\
-\frac{4 \gamma}{5} \\
\frac{2 \gamma}{15}
\end{array}\right), \\
p_{2} z_{2}=\left(\begin{array}{c}
\frac{2 \gamma}{15} \\
\frac{4 \gamma}{5} \\
\frac{2 \gamma}{15}
\end{array}\right) .
\end{gathered}
$$

Let us define the set $R_{1} b$ as follows:

$$
R_{1} b=\left\{\mathbf{z}^{(2)} \in \mathbb{R}^{3}: z_{1}^{(2)} \in\left[-\frac{2 \gamma}{15}, \frac{2 \gamma}{15}\right]\right\} .
$$

First, let us verify that the points in $R_{2}$ go to $R_{1} b$. The evaluation of the vector field in $p j_{1} z_{2}$ tells us that the spin is counterclockwise in $\mathbf{z}^{(2)}$ coordinates.

Consider the point $q_{2} z_{2}$ given by

$$
q_{2} z_{2}=\left(\begin{array}{c}
\frac{22 \gamma}{15}-\frac{4 \alpha}{3} \\
\frac{4 \gamma}{5} \\
\frac{7 \gamma}{15}-\frac{\alpha}{3}
\end{array}\right) .
$$

The angle produced by the radius from the point $q_{2} z_{2}$ to the $z_{1}(2)$ axis and the plane $z_{1}^{(2)}-z_{2}^{(2)}$ is given by

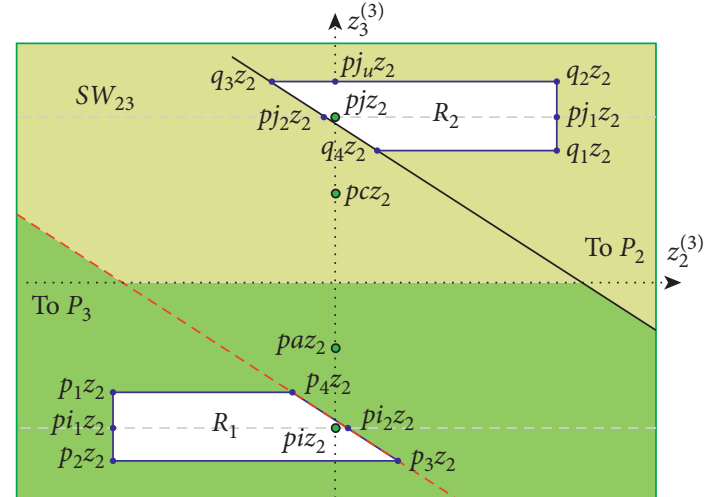

Figure 11: Regions $R_{1}$ and $R_{2}$ on the projection $z_{2}^{(2)}-z_{3}^{(2)}$.

$$
\arctan \left(\frac{(7 \gamma / 15)-(\alpha / 3)}{(4 \gamma / 5)}\right)=\arctan \left(\frac{7}{12}-\frac{5 \alpha}{12 \gamma}\right) .
$$

Under Assumption 1, the angle obeys the following inequality:

$$
\arctan \left(\frac{7}{12}-\frac{5 \alpha}{12 \gamma}\right) \leq \arctan \left(\frac{7}{12}\right) \leq 0.5281 .
$$

Consider then that the trajectory with an initial condition in $q_{2} z_{2}$ evolves for a duration that corresponds to $\pi-2(0.5281)=2.0854$. If after this duration the first component of the state vector $z_{1}^{(2)} \leq 2 \gamma / 15$ means that the trajectory with initial condition in $q_{2} z_{2}$ reaches $R_{1} b$. After this duration, $z_{1}^{(2)}$ is given by

$$
z_{1}^{(2)}=\left(\frac{22 \gamma}{15}-\frac{4 \alpha}{3}\right) e^{c(2.0854 / b)} .
$$

Under Assumption 1 for a big value of $z_{1}^{(2)}$ :

$$
\left(\frac{22 \gamma}{15}-\frac{4 \alpha}{3}\right) e^{c(2.0854 / b)} \leq\left(\frac{22 \gamma}{15}\right) e^{c(2.0854 / b)} \leq 0.0791 \gamma<\frac{2 \gamma}{15} .
$$

Thus, $q_{2} z_{2}$ reaches the region $R_{1} b$. Moreover, since the points in the segment $\overline{q_{2} z_{2} q_{1} z_{2}}$ produce a radius whose angle with the plane $z_{1}^{(2)}-z_{2}^{(2)}$ is between 0 and 0.5281 , the trajectories with the initial condition in this segment also reach the set $R_{1} b$.

Now consider the point $q_{3} z_{2}$

$$
q_{3} z_{2}=\left(\begin{array}{c}
\frac{22 \gamma}{15}-\frac{4 \alpha}{3} \\
\frac{2 c \gamma-17 a \gamma}{15 b}+\frac{a \alpha}{b} \\
\frac{7 \gamma-5 \alpha}{15}
\end{array}\right) .
$$

The angle of the radius at $q_{3} z_{2}$ with the plane $z_{1}^{(2)}-z_{2}^{(2)}$ is as follows: 


$$
-\arctan \left(\frac{((2 c \gamma-17 a \gamma) / 15 b)+a \alpha / b}{(7 \gamma-5 \alpha) / 15}\right)=-\arctan \left(\frac{2 c \gamma-17 a \gamma+15 a \alpha}{7 b \gamma-5 b \alpha}\right)
$$

Under Assumption 1, the angle should be less than

$$
\begin{aligned}
& -\arctan \left(\frac{2 c \gamma-17 a \gamma+15 a \alpha}{7 b \gamma-5 b \alpha}\right) \leq-\arctan \left(\frac{2 c}{6.5 b}-\frac{17 a}{6.5 b}+\frac{1.5 a}{6.5 b}\right) \\
& -\arctan \left(\frac{2 c}{6.5 b}-\frac{17 a}{6.5 b}+\frac{1.5 a}{6.5 b}\right) \leq-\arctan \left(-\frac{4}{6.5}-\frac{17}{6.5(25)}+\frac{1.5}{6.5(25)}\right)=0.6179 .
\end{aligned}
$$

To verify that the trajectory starting in $q_{3} z_{2}$ is not going to reach $\mathrm{SW}_{23}$ when the radius form an angle of $3 \pi / 2$ with the plane $z_{1}^{(2)}-z_{2}^{(2)}$, let us consider the following exaggerated scenario: The radius size corresponds to a duration equivalent to $10 \pi / 9$, but the $z_{1}^{(2)}$ component corresponds to a duration equivalent to $2 \pi / 3$ of oscillation, i.e., when the radius forms an angle of $3 \pi / 2$ with the plane $z_{1}^{(2)}-z_{2}^{(2)}$ a smaller radius than the real one is considered; also, a larger value of $z_{1}^{(2)}$ than the real value is considered.

Then to obtain the radius,

$$
r=e^{a(10 \pi / 9 b)} \sqrt{\left(\frac{2 c \gamma-17 a \gamma}{15 b}+\frac{a \alpha}{b}\right)^{2}+\left(\frac{7 \gamma-5 \alpha}{15}\right)^{2}} .
$$

Under Assumption 1 for the largest radius,

$$
\begin{gathered}
e^{a(10 \pi / 9 b)} \sqrt{\left(\frac{2 c \gamma-17 a \gamma}{15 b}+\frac{a \alpha}{b}\right)^{2}+\left(\frac{7 \gamma-5 \alpha}{15}\right)^{2}} \leq e^{a(10 \pi / 9 b)} \sqrt{\left(\frac{2 c \gamma-17 a \gamma}{15 b}\right)^{2}+\left(\frac{7 \gamma}{15}\right)^{2}} \\
e^{a(10 \pi / 9 b)} \gamma \sqrt{\left(\frac{2 c-17 a}{15 b}\right)^{2}+\left(\frac{7}{15}\right)^{2}} \leq 0.5984 \gamma
\end{gathered}
$$

The value of $z_{1}^{(2)}$ after the duration that corresponds to $2 \pi / 3$ is as follows:

$$
\left(\frac{22 \gamma}{15}-\frac{4 \alpha}{3}\right) e^{c(2 \pi / 3 b)}
$$

Under Assumption 1, for the biggest value of $z_{1}^{(2)}$

$$
\begin{aligned}
\left(\frac{22 \gamma}{15}-\frac{4 \alpha}{3}\right) e^{c(2 \pi / 3 b)} & \leq\left(\frac{22 \gamma}{15}\right) e^{c(2 \pi / 3 b)}, \\
\left(\frac{22 \gamma}{15}\right) e^{c(2 \pi / 3 b)} & \leq 0.07814 \gamma<\frac{2 \gamma}{15} .
\end{aligned}
$$

The points in $\mathrm{SW}_{23}$ fulfills the following equation:

$$
z_{3}^{(2)}=-(\gamma-\alpha)+z_{1}^{(2)}
$$

At this angle of $3 \pi / 2$ the radius is $r=-z_{3}^{(2)}$. Then, if the found values for this scenario fulfill the following inequality, it can be concluded that the trajectory with an initial condition in $q_{3} z_{2}$ does not reach $\mathrm{SW}_{23}$ after a duration that corresponds to an oscillation of $120^{\circ}$ :

$$
-.5984 \gamma>-(\gamma-\alpha)+0.07814 \gamma \text {. }
$$

Under Assumption 1 and the worst case,

$$
-.5984 \gamma>-\frac{9 \gamma}{10}+0.07814 \gamma=-0.82186 \gamma .
$$

Then, the trajectory remains for the duration that corresponds to $2 \pi / 3$. Furthermore, since $z_{1}^{(2)}<2 \gamma / 15$ the trajectory reaches $R_{1} b$. Since $q_{3} z_{2}$ is the point in the set $\left\{\mathbf{z}^{(2)} \in R_{2}: z_{2}^{(2)} \leq 0\right\}$ that produces the largest radius of that set, the same conclusion applies to the points in this set.

For the trajectories with the initial condition in the set $\left\{\mathbf{z}^{(2)} \in R_{2}: z_{2}^{(2)}>0\right\}$ the duration is equivalent to more than $2 \pi / 3$. Thus, the trajectories that start in $R_{2}$ reach the set $R_{1} b$.

Now to verify that the trajectories that start in $R_{2}$ reach $R_{1} \subset R_{1} b$ is enough to verify the trajectories starting in the 
segment $\overline{q_{1} z_{2} q_{2} z_{2}}$, since these produce the largest radius in $R_{1} b$.

Consider the points $q_{1} z_{2}$ and $q_{2} z_{2}$

$$
q_{1} z_{2}=\left(\begin{array}{c}
\frac{6 \gamma}{5}-\frac{4 \alpha}{3} \\
\frac{4 \gamma}{5} \\
\frac{\gamma}{5}-\frac{\alpha}{3}
\end{array}\right),
$$

The largest radius is at $q_{2} z_{2}$ while the smaller angle is at $q_{1} z_{2}$, then let us consider that radius of $q_{2} z_{2}$ with the angle of $q_{1} z_{2}$ and the end position at $3 \pi / 2$ with respect to the plane $z_{1}^{(2)}-z_{2}^{(2)}$, which is more than the possible rotation. The angle is given by

$$
\arctan \left(\frac{(\gamma / 5)-(\alpha / 3)}{(4 \gamma / 5)}\right)=\arctan \left(\frac{1}{4}-\frac{5 \alpha}{12 \gamma}\right)
$$

Under Assumption 1, the smallest angle is as follows:

$$
\arctan \left(\frac{1}{4}-\frac{5 \alpha}{12 \gamma}\right) \geq \arctan \left(\frac{5}{24}\right)=0.2054
$$

Thus for $3 \pi / 2-0.2054=4.507$

$$
\sqrt{\left(\frac{4 \gamma}{5}\right)^{2}+\left(\frac{7 \gamma}{15}-\frac{\alpha}{3}\right)^{2}} e^{a(4.507 / b)}
$$

Under Assumption 1 for the biggest radius,

$$
\begin{aligned}
\sqrt{\left(\frac{4 \gamma}{5}\right)^{2}+\left(\frac{7 \gamma}{15}-\frac{\alpha}{3}\right)^{2}} e^{a(4.507 / b)} & \leq \sqrt{\left(\frac{4 \gamma}{5}\right)^{2}+\left(\frac{7 \gamma}{15}\right)^{2}} e^{a(4.507 / b)}, \\
\sqrt{\left(\frac{4 \gamma}{5}\right)^{2}+\left(\frac{7 \gamma}{15}\right)^{2}} e^{a(4.507 / b)} & \leq \gamma \sqrt{\frac{193}{225}} e^{a(4.507 / b)} \leq 1.1091 \gamma .
\end{aligned}
$$

Consider the points $p_{1} z_{2}, p a_{1} z_{2}$, and $p_{2} z_{2}$

$$
\begin{aligned}
p_{1} z_{2}= & \left(\begin{array}{c}
\frac{2 \gamma}{15} \\
-\frac{4 \gamma}{5} \\
\alpha-\frac{13 \gamma}{15}
\end{array}\right), \\
p a_{1} z_{2}= & \left(\begin{array}{c}
0 \\
-\frac{4 \gamma}{5} \\
\alpha-\gamma
\end{array}\right), \\
p_{2} z_{2}= & \left(\begin{array}{c}
2 \gamma \\
-\frac{15}{4 \gamma} \\
-\frac{15}{5} \\
17 \gamma \\
15
\end{array}\right) .
\end{aligned}
$$
follows:

The minimum radius in the segment $\overline{p a_{1} z_{2}, p a_{2} z_{2}}$ is as

$$
\sqrt{\left(\frac{4 \gamma}{5}\right)^{2}+\left(\alpha-\frac{13 \gamma}{15}\right)^{2}}
$$

Under Assumption 1 for the smallest radius,

$$
\sqrt{\left(\frac{4 \gamma}{5}\right)^{2}+\left(\alpha-\frac{13 \gamma}{15}\right)^{2}} \geq \sqrt{\left(\frac{4 \gamma}{5}\right)^{2}+\left(\frac{23 \gamma}{30}\right)^{2}}=1.1081 \gamma
$$

$1.1092 \gamma \approx 1.1081 \gamma$, even when the angle of rotation was exaggerated, it is expected that the trajectories with an initial condition in $R_{1}$ reach $R_{2}$ or a self-excited attractor. In the same way, the trajectories with an initial condition in $R_{2}$ reach $R_{1}$ or go to a self-excited attractor.

Let us look at the system in $\mathbf{z}^{(2)}$ coordinates, as in the previous section with the previous switching surfaces, as $\gamma$ grows, $\mathrm{SW}_{23}$ and $p c z_{2}$ is further from the $\mathbf{z}_{\mathrm{eq} 2}^{*}$ and then it take more time for the trajectories close to $p c z_{2}$ to travel along the $z_{1}^{(2)}$ direction to get close to $\mathbf{z}_{\text {eq2 }}^{*}$. However, the expansion along $z_{2}^{(2)}$ and $z_{3}^{(2)}$ remains the same; then the subsets of initial conditions that reach the self-excited attractors without reaching $\mathrm{SW}_{23}$ again shrink in $I_{1}$ and $I_{2}$ but this time, as opposed to the previous case there exist a value of $\gamma$ such that the intersection disappear.

Then for a sufficiently big value of $\gamma$ we have a region $R_{1}$ such that any trajectory starting there remains crossing $R_{1}$ for $t>0$. Then, we could expect that exists either a periodic orbit, a hidden limit cycle, a hidden chaotic attractor, or a combination of the previous, which should go through $R_{1}$ and $R_{2}$. 


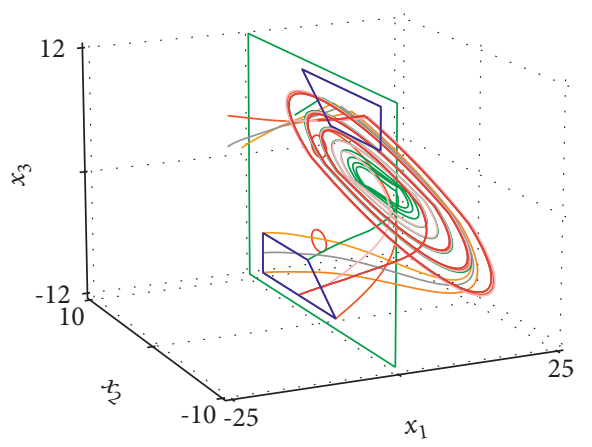

(a)

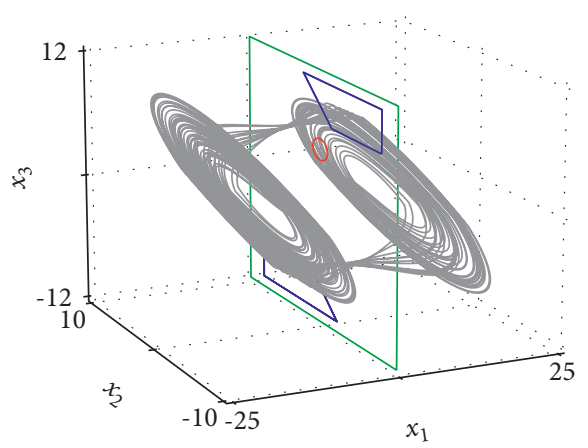

(b)

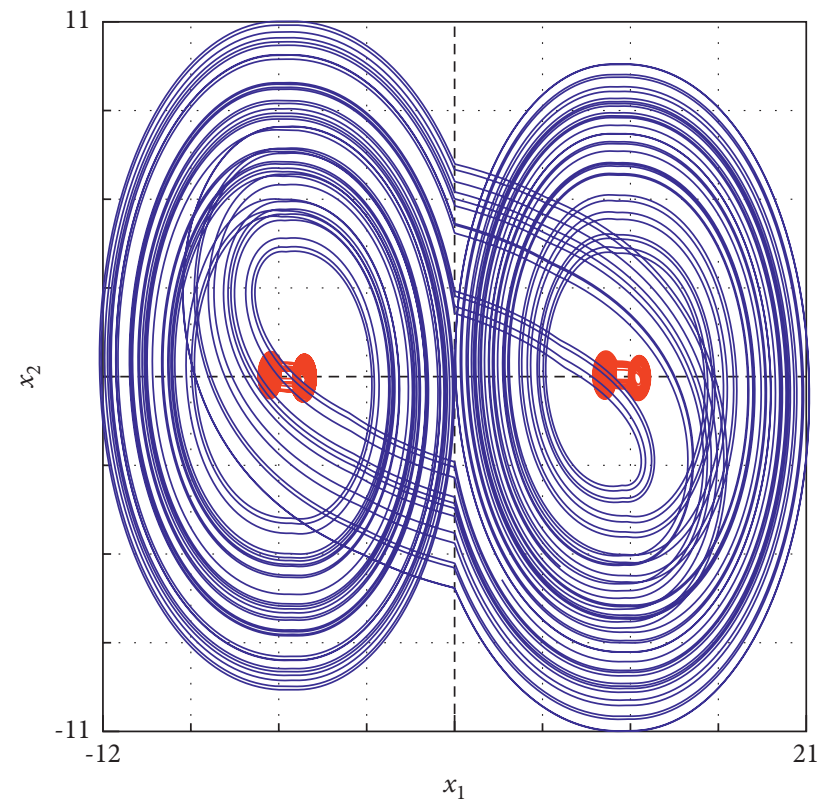

(c)

Figure 12: In (a), seven trajectories of the system given by (1), (3), (5), (13), and (90) starting in $R_{1}$ with $a=0.2, b=5, c=-7, \alpha=1$ and $\gamma=10$. In (b), hidden attractor for the same parameters and the initial condition $\mathbf{x}_{0}=(0,0,0)^{T}$ for $t \in[50000,50100]$. In (c), the projection of the self-excited attractors and the hidden attractor onto the plane $x_{1}-x_{2}$.

Also, as small differences in the initial conditions in $R_{1}$ could eventually produce a big separation of trajectories in $\mathrm{SW}_{23}$, sensitivity to initial conditions could also be expected. However, the formal proof is out of the scope of this work.

To verify the region for the parameters $a=0.2, b=5$, $c=-7, \alpha=1$ and $\gamma=10$ seven trajectories have been simulated and are shown in Figure 1(a).

The simulations of the two particular cases coincide with the conjecture. In Figure 12(b) it is shown the hidden attractor for the parameters $a=0.2, b=5, c=-7, \alpha=1$ and $\gamma=10$ and the initial condition $\mathbf{x}_{0}=(0,0,0)^{T}$ for $t \in[50000,50100]$. In Figure 1(c), it is shown the projection of the hidden attractor and the two self-excited attractors onto the plane $x_{1}-x_{2}$ for the same parameters.

\section{Conclusions}

In this work, an approach for the generation of multiscroll attractors was studied based on heteroclinic orbits. Particularly, we presented a quad-scroll self-excited attractor, which is split into two double-scroll self-excited attractor, so the system bifurcates from monostability to biestability. The approach is based on the coexistence of double-scroll selfexcited attractors surrounded the equilibria and presenting heteroclinic orbits. Increasing the distances between the double-scroll self-excited attractors generates a heterocliniclike orbit between the equilibria of two different doublescroll self-excited attractors. It is possible to generate hidden attractors surrounding the self-excited attractors by 
breaking the heteroclinic-like orbit. The study revealed a relationship between the existence of a hidden attractor and the trajectories that, when are seen on a larger scale resemble heteroclinic orbits which join the self-excited attractors. The findings suggest that new classes of multistable systems with a different number of self-excited and hidden attractors can be designed with a geometric approach.

\section{Data Availability}

The data used to support the findings of this study are included within the article.

\section{Disclosure}

A preprint of this study has previously been published (https://arxiv.org/abs/1908.03789).

\section{Conflicts of Interest}

There are no conflicts of interest regarding the publication of this paper.

\section{Acknowledgments}

R.J. Escalante-González is thankful to CONACYT for the scholarships granted. Eric Campos acknowledges CONACYT for the financial support through Project No. A1-S30433.

\section{References}

[1] G. A. Leonov, N. V. Kuznetsov, and V. I. Vagaitsev, "Localization of hidden Chua's attractors," Physics Letters A, vol. 375 , no. 23 , pp. $2230-2233,2011$.

[2] D. Dudkowski, S. Jafari, T. Kapitaniak, N. V. Kuznetsov, G. A. Leonov, and A. Prasad, "Hidden attractors in dynamical systems," Physics Reports, vol. 637, pp. 1-50, 2016.

[3] A. Anzo-Hernández, H. E. Gilardi-Velázquez, and E. Campos-Cantón, "On multistability behavior of unstable dissipative systems," Chaos: An Interdisciplinary Journal of Nonlinear Science, vol. 28, no. 3, Article ID 033613, 2018.

[4] M. A. Kiseleva, N. V. Kuznetsov, and G. A. Leonov, "Hidden attractors in electromechanical systems with and without equilibria," IFAC-PapersOnLine, vol. 49, no. 14, pp. 51-55, 2016.

[5] J. C. Sprott, "Some simple chaotic flows," Physical Review. E, Statistical Physics, Plasmas, Fluids, and Related Interdisciplinary Topics, vol. 50, no. 2, pp. R647-R650, 1994.

[6] W. G. Hoover, "Remark on "Some simple chaotic flows"” Physical Review A, vol. 51, no. 1, pp. 759-760, 1995.

[7] Z. Wei, "Dynamical behaviors of a chaotic system with no equilibria," Physics Letters A, vol. 376, no. 2, pp. 102-108, 2011.

[8] X. Wang and G. Chen, "Constructing a chaotic system with any number of equilibria," Nonlinear Dynamics, vol. 71, no. 3 , pp. 429-436, 2013.

[9] R. J. Escalante-González and E. Campos-Cantón, "Generation of chaotic attractors without equilibria via piecewise linear systems," International Journal of Modern Physics C, vol. 28, no. 01, Article ID 1750008, 2017.
[10] S. Jafari, J. C. Sprott, and S. M. R. Hashemi Golpayegani, "Elementary quadratic chaotic flows with no equilibria," Physics Letters A, vol. 377, no. 9, pp. 699-702, 2013.

[11] Z. Wang, S. Cang, E. O. Ochola, and Y. Sun, "A hyperchaotic system without equilibrium," Nonlinear Dynamics, vol. 69, no. 1-2, pp. 531-537, 2012.

[12] V.-T. Pham, "Sundarapandian vaidyanathan, christos volos, sajad jafari, and sifeu takougang kingni. A no-equilibrium hyperchaotic system with a cubic nonlinear term," Optik, vol. 127, no. 1, pp. 3259-3265, 2016.

[13] C. Li, J. C. Sprott, W. Thio, and H. Zhu, "A new piecewise linear hyperchaotic circuit," IEEE Transactions on Circuits and Systems II: Express Briefs, vol. 61, no. 12, pp. 977-981, 2014.

[14] F. R. Tahir, S. Jafari, V.-T. Pham, C. Volos, and X. Wang, "A novel no-equilibrium chaotic system with multiwing butterfly attractors," International Journal of Bifurcation and Chaos, vol. 25, no. 4, Article ID 1550056, 2015.

[15] J. A. K. Suykens, A. Huang, and L. O. Chua, "A family of n-scroll attractors from a generalized Chua's circuit," Archiv fur Elektronik und Ubertragungstechnik, vol. 51, no. 3, pp. 131-138, 1997.

[16] W. K. S. Tang, G. Q. Zhong, G. Chen, and K. F. Man, "Generation of N-Scroll attractors via sine function," IEEE Transactions on Circuits and Systems I: Fundamental Theory and Applications, vol. 48, no. 11, pp. 1369-1372, 2001.

[17] M. . E. Yalçin, J. A. K. Suykens, and J. Vandewalle, "Families of scroll grid attractors," International Journal of Bifurcation and Chaos, vol. 12, no. 1, pp. 23-41, 2002.

[18] B. Aguirre-Hernández, E. Campos-Cantón, J. A. LópezRenteria, and E. C. Díaz González, "A polynomial approach for generating a monoparametric family of chaotic attractors via switched linear systems," Chaos, Solitons \& Fractals, vol. 71, no. 1, pp. 100-106, 2015.

[19] E. Campos-Cantón, "Chaotic attractors based on unstable dissipative systems via third-order differential equation," International Journal of Modern Physics C, vol. 27, no. 1, Article ID 1650008, 2016.

[20] R. J. Escalante-González, E. Campos-Cantón, and M. Nicol, "Generation of multi-scroll attractors without equilibria via piecewise linear systems," Chaos: An Interdisciplinary Journal of Nonlinear Science, vol. 27, no. 5, Article ID 053109, 2017.

[21] X. Hu, C. Liu, L. Liu, J. Ni, and S. Li, "Multi-scroll hidden attractors in improved sprott a system," Nonlinear Dynamics, vol. 86, no. 3, pp. 1725-1734, 2016.

[22] S. Jafari, V.-T. Pham, and T. Kapitaniak, "Multiscroll chaotic sea obtained from a simple 3d system without equilibrium," International Journal of Bifurcation and Chaos, vol. 26, no. 02, Article ID 1650031, 2016.

[23] X. Hu, C. Liu, L. Liu, Y. Yao, and G. Zheng, "Multi-scroll hidden attractors and multi-wing hidden attractors in a 5dimensional memristive system," Chinese Physics B, vol. 26, no. 11, Article ID 110502, 2017.

[24] L. J. Ontañón-García and E. Campos-Cantón, "Widening of the basins of attraction of a multistable switching dynamical system with the location of symmetric equilibria," Nonlinear Analysis: Hybrid Systems, vol. 26, pp. 38-47, 2017.

[25] S. Ergun and S. Ozoguz, "Truly random number generators based on a double-scroll attractor," in Proceedings of the 2006 49th IEEE International Midwest Symposium on Circuits and Systems, vol. 1, pp. 322-326, Puerto Rico, Mexico, August 2006.

[26] M. García-Martínez, L. J. Ontañón-García, E. CamposCantón, and S. Čelikovský, "Hyperchaotic encryption based 
on multi-scroll piecewise linear systems," Applied Mathematics and Computation, vol. 270, pp. 413-424, 2015.

[27] X. Jin, X. Duan, H. Jin, and Y. Ma, "A novel hybrid secure image encryption based on the shuffle algorithm and the hidden attractor chaos system," Entropy, vol. 22, no. 6, 2020.

[28] R. J. Escalante-González and E. Campos, "Multistable systems with hidden and self-excited scroll attractors generated via piecewise linear systems," Complexity, vol. 2020, Article ID 7832489, 12 pages, 2020.

[29] R. J. Escalante-González and E. Campos-Cantón, "Coexistence of hidden attractors and self-excited attractors through breaking heteroclinic-like orbits of switched systems," arXiv, 2019, https://arxiv.org/abs/1908.03789.

[30] E. Campos-Cantón, J. G. Barajas-Ramirez, G. Solís-Perales, and R. Femat, "Multiscroll attractors by switching systems," Chaos, vol. 20, no. 1, Article ID 013116, 2010. 